\title{
European Respiratory Society guidelines for the management of children and adolescents with bronchiectasis
}

\author{
Anne B. Chang $\mathbb{1}^{1,2}$, Rebecca Fortescue ${ }^{3}$, Keith Grimwood ${ }^{4,5}$, Efthymia Alexopoulou $\mathbb{1}^{6}$, Leanne Bell ${ }^{7}$, \\ Jeanette Boyd ${ }^{8}$, Andrew Bush ${ }^{9}$, James D. Chalmers ${ }^{10}$, Adam T. Hill ${ }^{11}$, Bulent Karadag ${ }^{12}$, Fabio Midulla $\mathbb{( i )}^{13}$, \\ Gabrielle B. McCallum $\mathbb{( 1 0}^{2}$, Zena Powell ${ }^{7}$, Deborah Snijders ${ }^{14}$, Woo-Jung Song ${ }^{15}$, Thomy Tonia ${ }^{16}$, \\ Christine Wilson ${ }^{17}$, Angela Zacharasiewicz $\mathbb{1}^{18}$ and Ahmad Kantar (i) ${ }^{19}$
}

\begin{abstract}
${ }^{1}$ Dept of Respiratory and Sleep Medicine, Queensland Children's Hospital and Australian Centre for Health Services Innovation, Queensland University of Technology, Brisbane, Australia. ${ }^{2}$ Child Health Division, Menzies School of Health Research, Darwin, Australia. ${ }^{3}$ Population Health Research Institute, St George's University of London, London, UK. ${ }^{4}$ Depts of Infectious Disease and Paediatrics, Gold Coast Health, Southport, Australia. ${ }^{5}$ School of Medicine and Menzies Health Institute Queensland, Griffith University, Southport, Australia. ${ }^{6}$ 2nd Radiology Dept, National and Kapodistrian University of Athens, Attikon University Hospital, Athens, Greece. ${ }^{7}$ Bronchiectasis Paediatric Patient Advisory Group, European Lung Foundation, Alnwick, UK. ${ }^{8}$ European Lung Foundation, Sheffield, UK. ${ }^{9}$ Dept of Paediatric Respiratory Medicine, Royal Brompton Hospital and National Heart and Lung Institute, School of Medicine, Imperial College London, London, UK. ${ }^{10}$ College of Medicine, University of Dundee, Ninewells Hospital and Medical School, Dundee, UK. ${ }^{11}$ Dept of Respiratory Medicine, Royal Infirmary and University of Edinburgh, Edinburgh, UK. ${ }^{12}$ Division of Pediatric Pulmonology, Faculty of Medicine, Marmara University, Istanbul, Turkey. ${ }^{13}$ Dept of Maternal Science, Sapienza University of Rome, Rome, Italy. ${ }^{14}$ Dipartimento Salute della Donna e del Bambino, Università degli Studi di Padova, Padova, Italy. ${ }^{15}$ Dept of Allergy and Clinical Immunology, Asan Medical Center, University of Ulsan College of Medicine, Seoul, Korea. ${ }^{16}$ Institute of Social and Preventive Medicine, University of Bern, Bern, Switzerland. ${ }^{17}$ Dept of Physiotherapy, Queensland Children's Hospital, Brisbane, Australia. ${ }^{18}$ Dept of Pediatrics and Adolescent Medicine, Teaching Hospital of the University of Vienna, Wilhelminen Hospital, Klinikum Ottakring Vienna, Wien, Austria. ${ }^{19}$ Pediatric Asthma and Cough Centre, Istituti Ospedalieri Bergamaschi, University and Research Hospitals, Ponte San Pietro, Italy.
\end{abstract}

Corresponding author: Anne B. Chang (Anne.chang@menzies.edu.au)

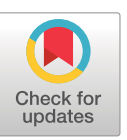

Copyright $\odot$ The authors 2021. For reproduction rights and permissions contact permissions@ersnet.org

This article has supplementary material available from erj.ersjournals.com

Received: 1 Aug 2020 Accepted: 21 Dec 2020
Shareable abstract (@ERSpublications)

Optimal management of children with bronchiectasis is important. This ERS evidence-based guideline, developed by a multidisciplinary team along with parents of children with bronchiectasis, aims to help clinicians optimise the management of bronchiectasis. https://bit.ly/ 3pvZ9Yi

Cite this article as: Chang AB, Fortescue R, Grimwood K, et al. European Respiratory Society guidelines for the management of children and adolescents with bronchiectasis. Eur Respir J 2021; 58: 2002990 [DOI: 10.1183/13993003.02990-2020].

\section{Abstract}

There is increasing awareness of bronchiectasis in children and adolescents, a chronic pulmonary disorder associated with poor quality of life for the child/adolescent and their parents, recurrent exacerbations, and costs to the family and health systems. Optimal treatment improves clinical outcomes. Several national guidelines exist, but there are no international guidelines.

The European Respiratory Society (ERS) Task Force for the management of paediatric bronchiectasis sought to identify evidence-based management (investigation and treatment) strategies. It used the ERS standardised methodology that included a systematic review of the literature and application of the GRADE (Grading of Recommendations Assessment, Development and Evaluation) approach to define the quality of the evidence and level of recommendations.

A multidisciplinary team of specialists in paediatric and adult respiratory medicine, infectious disease, physiotherapy, primary care, nursing, radiology, immunology, methodology, patient advocacy and parents of children/adolescents with bronchiectasis considered the most relevant clinical questions (for both clinicians and patients) related to managing paediatric bronchiectasis. 14 key clinical questions (seven PICO (Patient, Intervention, Comparison, Outcome) and seven narrative) were generated. The outcomes for each PICO were decided by voting by the panel and parent/patient advisory group.

This guideline addresses the definition, diagnostic approach and antibiotic treatment of exacerbations, pathogen eradication, long-term antibiotic therapy, asthma-type therapies (inhaled corticosteroids and 
bronchodilators), mucoactive drugs, airway clearance, investigation of underlying causes of bronchiectasis, disease monitoring, factors to consider before surgical treatment, and the reversibility and prevention of bronchiectasis in children/adolescents. Benchmarking quality of care for children/adolescents with bronchiectasis to improve clinical outcomes and evidence gaps for future research could be based on these recommendations.

\section{Scope and objectives}

This European Respiratory Society (ERS) guideline provides evidence-based recommendations for managing children and adolescents (aged $\leqslant 18$ years) with bronchiectasis unrelated to cystic fibrosis (CF). We focus on key management questions. Other important issues, such as environmental exposures, and rare cases of non-tuberculous mycobacterial (NTM) pulmonary disease in children/adolescents without CF are not addressed in this guideline.

The target audience is those involved in the care of children/adolescents with bronchiectasis, including specialists in respiratory medicine, infectious disease, paediatricians, thoracic surgeons, primary care physicians, pharmacists, respiratory physiotherapists, nurses, regulatory authorities, pharmaceutical companies and policy makers. The guideline also aims to inform adolescents and parents of children/ adolescents with bronchiectasis, which will assist discussions with healthcare teams and help facilitate access to appropriate care. However, as bronchiectasis is a complex disease with many causes, this guideline does not substitute for sound clinical judgement and requires appropriate adaptations to local circumstances (e.g. where tuberculosis prevalence is high). All recommendations should be interpreted according to the child/adolescent's circumstances, patients' perceptions, values and preferences, and the clinical setting.

\section{Introduction}

Bronchiectasis, a chronic pulmonary disorder, is an umbrella term for a clinical syndrome of recurrent or persistent wet/productive cough, airway infection and inflammation, and abnormal bronchial dilatation on chest computed tomography (CT) scans, which if detected early may be reversible over time with effective treatment $[1,2]$.

Bronchiectasis is no longer considered rare [1, 3, 4], but is one of the most neglected lung disorders [5], with high individual disease burden [6], economic cost [7], and poor quality of life (QoL) in children/ adolescents [8] and their parents [9]. Also, there are large disparities in the standards of care and outcomes between bronchiectasis and other chronic lung diseases [10], including in those with bronchiectasis from the same country [11].

Multiple risk and/or aetiological factors may lead to bronchiectasis in children/adolescents [1, 12]. Its prevalence shows geographical variation, but shares common features of chronic cough and recurrent exacerbations with lower airway infection/inflammation, which persist if left untreated. Interrupting the infection/inflammation cycle as early as possible with effective treatment is necessary to reverse and/or halt disease progression and further lung injury $[1,13]$. Indeed, bronchiectasis may be preventable in some children and thus their evaluation for possible treatable underlying causes is important $[1,12]$.

The pathophysiology of bronchiectasis is complex and poorly understood, with varying aetiologies and modifying factors [12]. These factors are likely dependent on the sampling frame studied (e.g. different aetiologies in different countries/settings). Nevertheless, the infection/inflammation paradigm, which is likely applicable to all aetiologies, involves airway infection causing inflammation, impaired mucociliary clearance and airway destruction, which in turn predisposes the damaged airway to further infection [12].

Exacerbations or "attacks" are particularly important in children/adolescents with bronchiectasis as they are associated with increased respiratory symptoms, impaired QoL [6], accelerated lung function decline $(-1.9$ forced expiratory volume in $1 \mathrm{~s}\left(\mathrm{FEV}_{1}\right)$ percent predicted per hospitalised exacerbation) [14], and high healthcare resource use [15] and costs (around EUR 20400 per hospitalisation in 2016) [7]. Importantly, patients and parents responding to the European Lung Foundation (ELF) survey rated exacerbations among the top three factors affecting their child's QoL. Thus, the impact on exacerbations is a dominant outcome measure when assessing the efficacy of interventions $[16,17]$.

Bronchiectasis in children/adolescents and adults has some similarities (e.g. wet/productive cough being the dominant symptom along with recurrent exacerbations), but there are also substantial differences. Children/adolescents require developmentally appropriate care, support and supervision from their parents. Mild radiographic bronchiectasis (bronchial dilatation) is reversible if treated optimally early, thereby 
avoiding the later deterioration in lung function [1]. In contrast, adults with untreated bronchiectasis symptoms from childhood have worse disease and poorer prognosis (compared with adult-onset bronchiectasis) [18]. Australian data indicate that $>60 \%$ of adults with bronchiectasis have symptoms from childhood [18]. Thus, early diagnosis is important, as is disease characterisation (e.g. defining exacerbations) and providing evidence-based management.

Furthermore, children/adolescents with bronchiectasis have different lower airway microbial profiles (bacterial pathogens [19] and microbial communities [20]), age-related immunological responses [21] and likely treatment outcomes [1]. Some diagnostic [1] and treatment methods also differ, e.g. airway clearance techniques, which are age- and cognition-dependent [22]. Moreover, aetiology and comorbidities can vary substantially between adults and children/adolescents [12].

Thus, the recent ERS [23] and British Thoracic Society [24] bronchiectasis guidelines were for adults only. The present guideline addresses this gap of an up-to-date international evidence-based guideline for managing children/adolescents with bronchiectasis unrelated to CF. It includes those with primary ciliary dyskinesia, where older ERS guidelines exist, but required updating [25, 26]. The objectives of managing children/adolescents with bronchiectasis are to: 1) optimise lung growth, 2) preserve lung function, 3) optimise QoL, 4) minimise exacerbations, 5) prevent complications and 6) if possible, reverse structural lung injury.

\section{Methods}

This guideline was developed by an ERS Bronchiectasis Task Force that included specialists in paediatric respiratory medicine with expertise in managing children/adolescents with bronchiectasis as well as paediatric experts in infectious disease, allergy immunology, radiology, physiotherapy and nursing, two global leaders in adult bronchiectasis, the Cochrane Airways Group coordinating editor (also a general practitioner), an ELF representative, bronchiectasis parent/patient advisory group (PAG) members and ERS methodologists. The ELF representative and two PAG representatives were full members of the Task Force and contributed to all recommendations. Conflicts of interest were declared at commencement of this project and prior to final submission, and managed in accordance with ERS policies. The specific expertise of the panel is outlined in the supplementary methods.

Between November 2018 and June 2020, the panel met 10 times (nine video-conferences and one face-to-face meeting) and a smaller methodology subgroup met a further 11 times online. The most relevant clinical questions on managing bronchiectasis in children/adolescents (for both clinicians and patients/parents) were discussed and agreed by the panel and PAG (figure 1). Following the ERS methodology [27], we formulated seven questions using the PICO (Patient, Intervention, Comparison, Outcome) format and seven narrative questions (NQs). The panel and the entire PAG voted on the outcomes of interest for each PICO a priori, based on their relative importance to children/adolescents

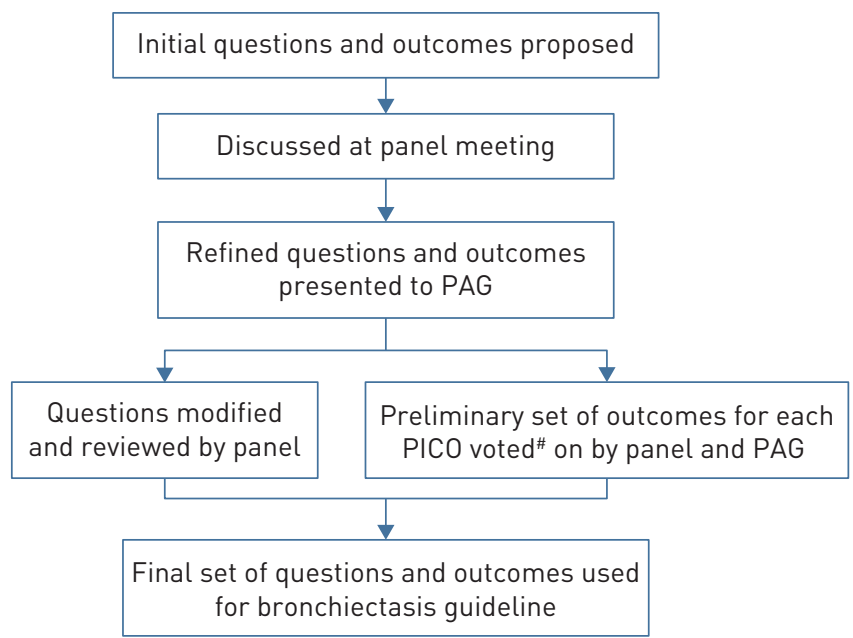

FIGURE 1 Schematic overview of the methodology used to develop the questions and outcomes used for this guideline. PAG: parent/patient advisory group. "*: using Survey Monkey (www.surveymonkey.com). 
with bronchiectasis and to clinical decision making. Following the GRADE (Grading of Recommendations Assessment, Development and Evaluation) approach [28], these outcomes were deemed as “critical”, "important" or "not important for clinical decision making" (the latter were then excluded from data extraction and further analysis), as listed in the evidence table for each PICO (refer to the supplementary methods). Systematic reviews were conducted to answer these questions. For NQs, systematic searches were conducted and evidence was reviewed in a narrative manner.

\section{Systematic reviews}

The Cochrane Airways Group information specialist designed and ran the search (supplementary material) for all questions. The initial searches undertaken in May 2019 were updated in April 2020. Results of the search were sent to panel member pairs and R.F. or A.B.C. Searches were independently screened by at least two people using inclusion and exclusion criteria determined by the Task Force (supplementary methods). A PRISMA (Preferred Reporting Items for Systematic Reviews and Meta-Analyses) diagram was generated for each question (supplementary figures). For selected PICOs, we undertook additional searches to seek supportive evidence from the literature, including the CF literature (described in the supplementary methods) for a narrative review of supportive evidence when the panel considered it was important to undertake this additional task. Articles were summarised using the ERS framework for guideline development, including both systematic (for PICOs) and pragmatic/narrative (for NQs) reviews of the evidence [27].

\section{Assessing the level of evidence and degree of recommendations}

Evidence summary tables and evidence to decision (EtD) frameworks were generated for each PICO, while only EtD frameworks were generated for NQs (supplementary material). For NQs, in accordance with the updated ERS methodologies [27], the approach was narrative; that for the evidence was a partial narrative approach (i.e. we did not undertake meta-analysis, but did include numbers). These were used by the panel to formulate recommendations and strength by consensus and/or voting. In accordance with ERS requirements [27], we used GRADE [29] to assess the confidence in the evidence (quality) and strength of the recommendations. The recommendations are graded as strong or conditional with key considerations summarised in table 1. In line with GRADE [29], the terms "we recommend" are used for strong recommendations and "we suggest" for conditional recommendations. Opinions of patients/parents of children/adolescents were captured from: a) two parents participating in discussions on every

TABLE 1 GRADE-based recommendations used in this document, based on GRADE [29] and used in accordance with the European Respiratory Society methodology [27]

\begin{tabular}{|c|c|c|}
\hline Target group & Strong recommendations ${ }^{\#}$ & Conditional (weak) recommendations \\
\hline $\begin{array}{l}\text { Patients/carers } \\
\text { and their } \\
\text { parents }\end{array}$ & $\begin{array}{l}\text { All or almost all informed people would } \\
\text { follow the recommended advice for or } \\
\text { against an intervention. They would } \\
\text { request the recommended intervention if } \\
\text { it is not offered. }\end{array}$ & $\begin{array}{l}\text { Most informed people would choose the } \\
\text { recommended course of action, but many } \\
\text { would not. }\end{array}$ \\
\hline Clinician & $\begin{array}{l}\text { Most carers/patients should follow the } \\
\text { recommended advice for or against an } \\
\text { intervention. }\end{array}$ & $\begin{array}{l}\text { Recognise that different choices will be } \\
\text { appropriate for different carers/patients in } \\
\text { different circumstances. Clinicians and } \\
\text { other healthcare providers must be } \\
\text { cognisant of the need to devote more } \\
\text { time for shared decision making by which } \\
\text { the carers/patients ensure that the } \\
\text { informed choice reflects individual values } \\
\text { and preferences. }\end{array}$ \\
\hline Policy r & $\begin{array}{l}\text { The recommendation can be adopted as a } \\
\text { policy in most situations. }\end{array}$ & $\begin{array}{l}\text { Policy making will require substantial debate } \\
\text { and involvement of many stakeholders. }\end{array}$ \\
\hline \multicolumn{3}{|c|}{$\begin{array}{l}\text { Recommendations are graded as strong or conditional after considering the quality of the evidence, the } \\
\text { balance of desirable and undesirable consequences of compared management options, the assumptions about } \\
\text { the relative importance of outcomes, the implications for resource use, and the acceptability and feasibility of } \\
\text { implementation [134]. Strong recommendations are worded as "we recommend", while conditional } \\
\text { recommendations are worded as "we suggest". ": while strong recommendations are generally based on high- } \\
\text { or moderate-quality evidence, applicable to most patients for whom these recommendations are made, they } \\
\text { may not apply to all patients in all settings. No recommendations can address all of the unique features of } \\
\text { individual patients and clinical circumstances. }\end{array}$} \\
\hline
\end{tabular}


recommendation and b) the ELF survey undertaken in 2019-2020 on the priorities and needs of parents whose children/adolescents have bronchiectasis or adults with bronchiectasis as a child/adolescent.

\section{Results}

PICO/NQ PRISMA diagrams (supplementary figures) depict the number of studies identified and selected for each question. The EtD frameworks for all questions (complete versions in the supplementary material) are summarised in the following and grouped into clinically relevant topics (diagnosis, evaluating the cause, defining exacerbations, management, monitoring, and reversibility and prevention).

\section{Diagnosis}

\section{PICO Question .}

In children/adolescents suspected of bronchiectasis: a) Should multidetector chest computed tomography (MDCT) scans with high-resolution CT (HRCT) be used instead of conventional HRCT alone for diagnosing bronchiectasis? b) What CT criteria for broncho-arterial dilatation (BAR) should be used?

\section{Recommendations}

- In children/adolescents suspected of bronchiectasis, we suggest that high-resolution MDCT scans with HRCT be used instead of conventional HRCT to diagnose bronchiectasis in children/adolescents. (Conditional recommendation, very low quality of evidence.)

- In children/adolescents suspected of bronchiectasis, we suggest that paediatric-derived BAR (defined by the ratio of the inner diameter of the airway to the outer diameter of the adjacent artery) of $>0.8$ is used to define abnormality instead of the adult cut-off of $>1-1.5$. (Conditional recommendation, very low quality of evidence stemming from the narrative review.)

\section{Summary of evidence}

No direct evidence in children/adolescents was available. Two non-blinded observational studies in adults [30, 31] reported MDCT scans (contiguous helical scan with $1 \mathrm{~mm}$ collimation) were superior at detecting and determining the extent of bronchiectasis, compared to conventional HRCT (1 mm collimation at 10 $20 \mathrm{~mm}$ intervals [30]). Using high-resolution MDCT as the gold standard, the sensitivity of conventional HRCT for diagnosing the number of patients with bronchiectasis was 96\% (95\% CI 90-98\%) and specificity was 69\% (95\% CI 54-81\%). Sensitivity and specificity for detecting the number of lobes with bronchiectasis was 89\% (95\% CI 84-92\%) and 81\% (95\% CI 78-84\%), respectively (evidence table in the supplementary material).

BAR correlates with age in adults without cardio-respiratory problems [32]. Our narrative summary of evidence includes two studies in children/adolescents [33, 34] without lower airway disease. Both [33, 34] found the mean \pm SD BAR is significantly lower in children/adolescents $(0.63 \pm 0.07$ in children/adolescents versus $0.70 \pm 0.1$ in adults) and the mean $+2 \mathrm{sD}=0.77$ (the upper limit of normal, rounded up to 0.8 ) [33].

Other supportive evidence

The narrative evidence depicts the impact of diagnosing bronchiectasis, particularly when diagnosed early. Treatment in children/adolescents post-radiographic diagnosis of bronchiectasis can stabilise, or even improve, lung function in heterogenous patient cohorts [14, 35, 36], including those with immunodeficiency [37]. One study [38] reported early diagnosis of bronchiectasis was important for improving QoL.

\section{Justification of recommendation}

This recommendation places a relatively higher value on more accurate and early detection of bronchiectasis and its importance on subsequent management, and a relatively lower value on evidence directness and quality. It is widely accepted that HRCT is the radiographic gold standard for confirming bronchiectasis. Many types of CT scanners are currently available and will continue to improve with greater precision and less radiation for patients. Adult-derived data (evidence table in the supplementary material) showed MDCT detects more cases of bronchiectasis than conventional HRCT. However, no paediatric data exist currently. The narrative summary provided circumstantial evidence that diagnosing bronchiectasis changes management, and optimal management stabilises or improves lung function, reduces exacerbations and improves QoL.

The early diagnosis of bronchiectasis was one of the top priorities articulated by parents of children/ adolescents with bronchiectasis or adults who had bronchiectasis as a child in the ELF survey. As BAR correlates with age [32] and increases as bronchiectasis becomes more severe (from cylindrical to varicose 
to cystic [1]), we suggest clinicians use a lower threshold in children/adolescents (BAR $>0.8$ ) to define abnormality when suspecting bronchiectasis.

\section{Implementation considerations}

CT scans need to be performed promptly to diagnose bronchiectasis early and there is a need to develop strategies to improve 1) availability and access to high-quality scanners that reduce radiation exposure, and 2) interpretation of paediatric chest CT scans. Using the suggested paediatric-defined BAR threshold of 0.8 may result in more radiographic-based diagnoses of bronchiectasis in children with chronic wet cough and reduce problems of drug reimbursement in some countries. However, as there are false positives with diagnosing bronchiectasis based purely on BAR, the panel advocated that BAR alone should not be used to diagnose bronchiectasis, i.e. it is best based on the presence of clinical features consistent with this diagnosis and confirmed radiographically.

\section{Evaluating the cause}

\section{Narrative Question 1}

In children/adolescents with suspected or confirmed bronchiectasis, what standard tests that impact on clinical outcomes should be undertaken when managing this group of patients?

\section{Recommendations}

- In children/adolescents with suspected or confirmed bronchiectasis, we suggest they have a minimum panel of tests undertaken, as done currently by most experts in the field. (The minimum panel of tests comprises: 1) chest CT scan (to diagnose bronchiectasis), 2) sweat test, 3) lung function tests (in children/adolescents who can perform spirometry), 4) full blood count, 5) immunological tests (total IgG, IgA, IgM, IgE and specific antibodies to vaccine antigens) and 6) lower airway bacteriology.) (Conditional recommendation, very low quality of evidence stemming from the narrative review.)

- In selected children/adolescents with bronchiectasis, we suggest additional tests are considered based on their clinical presentation. These include additional in-depth immunological assessments (in consultation with a paediatric immunologist), diagnostic bronchoscopy with bronchoalveolar lavage analysis (microbiology), and tests for airway aspiration, primary ciliary dyskinesia and gastro-oesophageal reflux disease. (Conditional recommendation, low quality of evidence stemming from the narrative review.)

Remarks: In settings where tuberculosis or HIV has a high prevalence and/or there is a history of close contact with tuberculosis, assessment for tuberculosis infection/disease or HIV, respectively, is also undertaken as part of the minimum panel of tests.

\section{Summary of evidence}

We identified 21 studies; all were observational studies. Of these studies, 18 were retrospective and three were prospective (supplementary material). Two [39, 40] of the three prospective studies [39-41] reported diagnostic yields for some tests. Nevertheless, several investigations were undertaken consistently (minimum panel) by experts in the field. From these tests, the aetiology of bronchiectasis varied (34$86 \%)$. In the two studies that reported specifically on diagnostic yields, immunology evaluation provided a diagnosis in 42\% [42] and bronchoscopy with bronchoalveolar lavage gave useful information in 12-41\% [40, 42].

\section{Justification of recommendation}

A conditional recommendation was selected based on the large desirable effect and likely trivial undesirable effects of setting a standard set of investigations as well as the risk and harm of not managing common or critical conditions related to bronchiectasis in children/adolescents. Finding causes of bronchiectasis was one of the research priorities identified by the PAG and the ELF survey. Lung function and respiratory cultures are part of minimum assessment. Although they do not identify the cause, these tests help assess severity and guide antibiotic choices, thus optimising treatment.

\section{Implementation considerations}

Identifying the aetiology has management implications (e.g. specific treatment for immunodeficiency, genetic causes for future family planning, etc.). Health services should increase accessibility to centres practising standard-of-care management for children/adolescents with bronchiectasis that includes undertaking the recommended minimum panel of tests. 


\section{Recommendations}

For clinical purposes:

- In children/adolescents with bronchiectasis, we suggest that a respiratory exacerbation is considered present when a child/adolescent has increased respiratory symptoms (predominantly increased cough with or without increased sputum quantity and/or purulence) for $\geqslant 3$ days. (Conditional recommendation, low quality of evidence stemming from the narrative review.)

Remarks: Other important but less common respiratory symptoms such as haemoptysis, chest pain, breathlessness and wheeze may not be present. Clinicians should not rely on changes in chest auscultation findings and chest radiographs to diagnose an exacerbation as, although important, these findings are not always present. Systemic symptoms (fever, fatigue, malaise, and change in child's behaviour and appetite) may also herald onset of an exacerbation, but are non-specific. Blood markers (e.g. elevated C-reactive protein, neutrophilia and interleukin-6) provide supportive evidence of the presence of an exacerbation. However, these indices are less important in defining exacerbations, but are likely useful for research purposes. Also, markers such as interleukin-6 are not standard clinical tests.

- In children/adolescents with bronchiectasis, we recommend that the presence of dyspnoea (increased work of breathing) and/or hypoxia should be considered a severe exacerbation, irrespective of duration. (Strong recommendation, low quality of evidence stemming from the narrative review.)

\section{Summary of evidence}

We identified 13 paediatric papers and one adult-based consensus document [16]. Of the paediatric-focused papers, two were defined within the published protocols [43, 44] (with the corresponding randomised controlled trials (RCTs) published $[45,46]$ ) using antibiotics at the onset of an exacerbation and three were published RCTs $[17,47,48]$ where exacerbations were outcomes. Two cohort studies (one prospective [49] and one retrospective [50]) specifically evaluated exacerbation definitions. Four papers were related solely to primary ciliary dyskinesia (one retrospective review [51], one protocol [52] that was published after the latest search [53], and two consensus-derived descriptions [54, 55] for children/adolescents and adults with primary ciliary dyskinesia, which differed substantially from one another).

While there are some similarities, overall the definitions used in these studies varied widely (e.g. defining an exacerbation for initiating antibiotics can be different to when it is used as an outcome measure for RCTs).

\section{Other supportive evidence}

The adult-derived consensus definition for research (i.e. not for clinical use) was framed around a deterioration in three or more symptoms (cough, sputum volume and/or consistency, sputum purulence, breathlessness and/or exercise tolerance, fatigue and/or malaise, and haemoptysis) for $\geqslant 48 \mathrm{~h}$. The definition also required "a change in treatment” [16].

\section{Justification of recommendation}

The recommendation was based upon several prospective studies and evidence that parents value recognising and treating respiratory exacerbations early. We considered that at least 3 days of increased symptoms are required for the definition, except when immunodeficiency or hypoxia/dyspnoea are present. For those with immunodeficiency, a lower threshold is suggested (as commencing treatment earlier may be required). No timeframe is required for those with hypoxia/dyspnoea as immediate treatment is mandated as there is a risk of acute deterioration and death.

\section{Implementation considerations}

Managing exacerbations is a key component of bronchiectasis care and one of the three top issues for parents. Thus, it is important to increase patient, parent/carer and health professional education in recognising exacerbations and commencing additional treatments.

Also, children/adolescents with neurodevelopmental conditions may have subtle and/or individually recognised symptoms of an exacerbation, whereby earlier treatment may be necessary.

\section{Management}

\section{Airway clearance}

PICO Question 4

In children/adolescents with bronchiectasis, should regular airway clearance techniques (ACT) (compared to no ACT) be undertaken? Subgroup analyses for a) short- versus long-term and b) stable versus exacerbation states. 
Recommendation

- In children/adolescents with bronchiectasis, we recommend they are taught and receive regular ACT or manoeuvres. (Strong recommendation, low quality of evidence.)

Remarks: Individualised ACT that are development- and age-appropriate are best taught by a paediatric-trained chest physiotherapist (figure 2). The frequency of ACT is best individualised. As children/adolescents mature, techniques may need to be changed, and thus the ACT type and frequency is best reviewed at least biannually by physiotherapists with expertise in paediatric respiratory care. During acute exacerbations of bronchiectasis, children/adolescents should receive ACT more frequently.

\section{Summary of evidence}

We identified one small ( $\mathrm{n}=24)$ RCT in children/adolescents [56] and two RCTs [57, 58] in adults (supplementary material). The paediatric study [56] that compared 1-month hospital-supervised, personalised ACT with unsupervised therapy at home (we equated this to controls without effective treatment) described a better median $\mathrm{FEV}_{1} \%$ pred in the intervention group (86.3\%) versus controls (68.8\%) at 1 month and 1 year (86.0\% versus 69.3\%). All three RCTs showed consistent improvement in lung function. For other critical outcomes, data were lacking in children/adolescents. Data from the adult-based RCTs [57, 58] (evidence table in the supplementary material) showed consistent results with improved QoL indices and sputum volume with ACT (versus no ACT), but no significant difference in the number of exacerbations (despite favouring ACT).

Additional evidence from adults (included here as mentioned in the methods and supplementary material): the benefits of ACT are supported by recent systematic reviews [23, 24, 59-61] of studies in adults

Infant

Toddler

\section{Child}

Modified GAD or GAD

Chest percussion with/without expiratory vibration

Positioning

Chest percussion with/without expiratory vibration

\begin{tabular}{|c|c|c|}
\hline \multirow{3}{*}{$\begin{array}{l}\text { Expiratory } \\
\text { flow } \\
\text { modification }\end{array}$} & & Blowing games \\
\hline & Assisted autogenic & Forced expirations, huffing, active cycle of breathing technique \\
\hline & drainage & Autogenic drainage \\
\hline
\end{tabular}

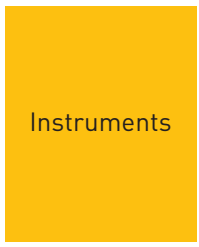

PEP via bottle, mouthpiece or mask
Oscillating PEP devices with/without nebuliser
High-frequency chest wall oscillation ("vest" therapy)
Oscillating PEP with forced expiration technique

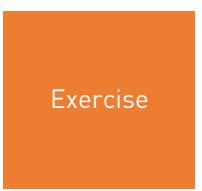

Bouncing on a fitball (supported/unsupported)

Vigorous activity (including active video games), physical exercise Vertical acceleration activities, e.g. trampoline

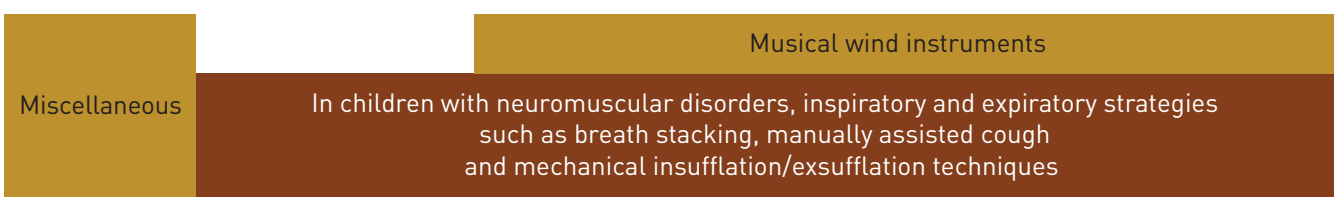

FIGURE 2 There are many different airway clearance techniques; in children/adolescents, these are age-specific and best taught by physiotherapists experienced in managing children/adolescents with bronchiectasis. GAD: gravity-assisted drainage; PEP: positive expiratory pressure. 
(no available meta-analyses of data), but with very-low- to low-level evidence. One systematic review of acute exacerbations found six adult-based studies involving 120 people, but none included a "no treatment" group [61]. The authors reported ACT during acute exacerbations resulted in no adverse events, improved sputum clearance, and a non-statistically significant improvement in lung function and symptoms [61].

Other supportive evidence

Three recent CF-related systematic reviews [62-64] provided data supporting ACT, and one study [65] described significant declines in lung function ( $\mathrm{FEV}_{1}$ and forced vital capacity (FVC) \% pred) without 3 weeks of ACT and improved lung function following its recommencement.

Justification of recommendation

Although the evidence for ACT improving clinical outcomes is very low, a strong recommendation was selected based on moderate desirable and trivial but time-consuming undesirable effects for undertaking ACT and the risk of harm if ACT is not undertaken. Where data exist, results are consistent and favour ACT compared with controls. Also, the panel and PAG described the use of ACT as a key intervention and one that is universally advocated for children/adolescents with bronchiectasis.

As there are many ACT methods, and developmental stage and cognitive abilities vary widely between children/adolescents (0-18 years), individualised therapy taught, and reviewed at least biannually, by paediatric-trained chest physiotherapists (figure 2) is recommended. Exacerbations increase airway secretions and enhancing their clearance would be beneficial.

Implementation considerations

Individualised ACT that are development- and age-appropriate are best taught by paediatric-trained chest physiotherapists (figure 2). Access to paediatric-trained physiotherapists was raised by the PAG. Adherence to the prescribed regime, especially over prolonged periods, is challenging. Also, the frequency and best ACT method(s) remain uncertain. Adjustment to the type of ACT during exacerbations may be necessary (e.g. exercises may not be feasible).

\section{Mucoactive agents}

PICO Question 3

In children/adolescents with bronchiectasis, should mucoactive agents (compared to no mucoactive agents) be used routinely? Subgroup analyses for a) short- versus long-term, b) stable versus exacerbation states and c) type of mucoactive agent.

\section{Recommendations}

- In children/adolescents with bronchiectasis, we recommend that recombinant human DNase (rhDNase) is not used routinely. (Strong recommendation, very low quality of evidence.)

- In children/adolescents with bronchiectasis, we suggest that bromhexine is not used routinely. (Conditional recommendation, very low quality of evidence.)

- In children/adolescents with bronchiectasis, we suggest that neither inhaled mannitol nor hypertonic saline are used routinely. (Conditional recommendation, very low quality of evidence.)

Remarks: Inhaled mannitol or 6-7\% hypertonic saline may be considered in selected patients, e.g. those with high daily symptoms, frequent exacerbations, difficulty in expectoration and/or poor QoL. If well tolerated, the use of hypertonic saline or mannitol could improve the QoL and facilitate expectoration. For hypertonic saline and mannitol, children should be old enough to tolerate these interventions, and the panel also considered that short-acting $\beta_{2}$-agonists should be used prior to inhaling either hypertonic saline or mannitol. The first dose of hypertonic saline or mannitol should be administered under medical supervision. The substantially higher cost of mannitol compared with hypertonic saline should also be taken into consideration.

Summary of evidence

We identified only adult-based RCTs involving rhDNase $(n=2[66,67])$, hypertonic saline $(n=3$ [68-70]), mannitol ( $n=2[71,72])$ and bromhexine $(n=1$ [73]). Quality of evidence was very low to low, depending on the intervention

Regular rhDNase for 24 weeks (compared with placebo) significantly increased exacerbation rates (relative risk 1.35, 95\% CI 1.01-1.79), and worsened FEV F $_{1}$ and FVC [66]. Data from the smaller and shorter RCT [67] were consistent, but could not be combined with the larger study (supplementary material). rhDNase was also associated with increased hospitalisation and adverse events. Studies using mannitol failed to meet their primary end-point, but mannitol significantly improved some QoL subdomains and sputum 
volume, and prolonged time-to-next exacerbation. The effect of hypertonic saline was like mannitol; however, data from RCTs could not be combined. Although the small study on bromhexine favoured its use for sputum volume and $\mathrm{FEV}_{1}$, there were more adverse events (OR 2.93, 95\% CI 0.12-73.97). See the evidence table in the supplementary material.

\section{Justification of recommendation}

The panel considered that the overall weight of the literature, combined with biological plausibility, would lead most clinicians to be very concerned about using rhDNase due to the potential adverse effects. Although the quality of the evidence for rhDNase is very low, there is risk of substantial harm (increased risk of exacerbations and faster lung function decline). The panel also considered that the overall weight of the literature would lead most clinicians to be very concerned about using bromhexine due to the potential adverse effects. Thus, the balance of the evidence favours not using rhDNase and bromhexine routinely based on patient/parents values, the substantial adverse effects described above and the lack of efficacy of these treatments.

The balance probably favours administering hypertonic saline and mannitol in some patients. For example, in adults, mannitol (compared with controls) was beneficial (significantly fewer exacerbations, prolonged time-to-next exacerbation and symptomatic improvement) in the subgroup with a high symptom burden [74].

Implementation considerations

Health professionals should be warned of the potential harmful effects of rhDNase. For hypertonic saline and mannitol, children should be old enough to tolerate these interventions with pre-inhalation of a SABA. Education on using these medications and equipment care is also needed.

Use of antibiotics

PICO Question 5

In children/adolescents with bronchiectasis, should systemic courses of antibiotics (compared to no antibiotics) be used to treat an acute respiratory exacerbation (type and duration)?

Recommendation

- In children/adolescents with bronchiectasis and an acute respiratory exacerbation, we recommend a systemic course of an appropriate antibiotic is used for 14 days. (Strong recommendation, moderate quality of evidence.)

Remarks: The empiric antibiotic of choice is amoxicillin-clavulanate, but the type of antibiotics chosen should be based on the patient's airway cultures (e.g. those with Pseudomonas aeruginosa require different treatment regimens to those without) and history of antibiotic hypersensitivity reactions. When the exacerbation is severe (e.g. the child/adolescent is hypoxic) and/or when the child/adolescent does not respond to oral antibiotics, intravenous antibiotics will be needed.

Summary of evidence

The evidence summary shows a single high-quality RCT supporting antibiotics for treating exacerbations. In that trial [45], amoxicillin-clavulanate was superior to placebo at resolving symptoms after 14 days treatment. Azithromycin was associated with improvement, but did not reach statistical significance of superiority over placebo. Amoxicillin-clavulanate also significantly reduced exacerbation duration, while this was similar between azithromycin and placebo among those whose symptoms resolved by day 14 [45]. No between-group differences were detected for time-to-next exacerbation, QoL or hospitalisations, although hospitalisation was uncommon in all groups [45]. The optimal duration of treatment with antibiotics is yet to be studied.

Other supportive evidence

Although no comparable placebo-controlled RCTs in adults exist, recommendations in adult guidelines $[23,24]$ are similar. Also, antibiotic treatment for acute exacerbations of bronchiectasis is considered standard of care.

\section{Justification of recommendation}

Our strong recommendation is based on a single high-quality RCT in children/adolescents and extensive clinical experience. Exacerbation resolution and duration both showed a benefit from the intervention. Importantly, the trial did not detect an increase in adverse events in the antibiotic treatment groups compared to placebo, although such events were uncommon. 
An earlier RCT, which did not meet the inclusion criteria [46], comparing amoxicillin-clavulanate to azithromycin for treating non-severe exacerbations found that by day 21 azithromycin was non-inferior to amoxicillin-clavulanate (within 20\% margin). However, symptom resolution in those receiving azithromycin took a median 4 days longer than those receiving amoxicillin-clavulanate, a statistical and clinically significant result [46].

Implementation considerations

Patients should have access to appropriate antibiotics for the recommended duration of treatment.

PICO Question 7

In children/adolescents with bronchiectasis and recurrent exacerbations, should long-term ( $\geqslant 2$ months) antibiotics (compared to no antibiotics) be used to reduce exacerbations?

Recommendation

- In children/adolescents and adolescents with bronchiectasis and recurrent exacerbations, we recommend treatment with long-term macrolide antibiotics to reduce exacerbations. (Strong recommendation, low quality of evidence.)

Remarks: Based on the panel's experience, we suggest long-term macrolide antibiotics only in those who have had more than one hospitalised or three or more non-hospitalised exacerbations in the previous 12 months. Such a course should be for at least 6 months with regular reassessment to determine whether the antibiotic continues to provide a clinical benefit. Children/adolescents receiving longer treatment courses (>24 months) should continue to be evaluated for risk versus benefit. This suggestion is in the context of lacking data concerning when long-term azithromycin should be initiated, and the need for caution because of increasing antibiotic resistance among bacterial pathogens within patients and the community. While NTM are very rarely detected in children/adolescents with bronchiectasis, we suggest a lower airway specimen is obtained (when possible) to exclude their presence before commencing long-term macrolide antibiotics. We encourage strategies to ensure adherence to the macrolide regimen as $\geqslant 70 \%$ adherence improves efficacy and reduces antibiotic resistance.

Summary of evidence

There were three RCTs $[17,47,48]$ and the combined data showed that macrolides reduced the number of children/adolescents experiencing any exacerbations during the trial period (relative risk 0.86, 95\% CI 0.75-0.99). Of these RCTs, one involved only children/adolescents with HIV [48] and it was a small study that found no effect. The largest of the RCTs described that using long-term azithromycin halves the frequency of exacerbations (incidence rate ratio 0.50 , 95\% CI $0.35-0.70$ ) and also likely reduces hospitalisation $(\mathrm{p}=0.06)$ [17].

There was no significant difference in serious adverse events when azithromycin was used compared to placebo. Indeed, serious adverse events were numerically lower in the azithromycin group (relative risk 0.57, 95\% CI 0.31-1.05). However, there were significant increases in macrolide-resistant bacteria in the upper airways (nasopharyngeal swabs) in those receiving long-term azithromycin compared to placebo.

Other supportive evidence

Although the single high-quality study was undertaken in Indigenous children/adolescents, the efficacy of macrolides at reducing exacerbations is consistent. Meta-analysis examining the efficacy of macrolides in adults with bronchiectasis shows similar effects (relative risk of being exacerbation-free when taking azithromycin compared with placebo 1.66, 95\% CI 1.37-2.02 in adults [75]). Studies in adults were substantially shorter in duration (6-12 months versus up to 24 months in the main paediatric RCT [17]). A recent RCT on azithromycin in adults with primary immunodeficiency and previous respiratory exacerbations (85\% had bronchiectasis) also showed similar results (compared with placebo, hazard ratio (HR) for exacerbation 0.5, 95\% CI 0.3-0.9; p=0.03; for hospitalisation HR 0.5, 95\% CI 0.2-1.1; p=0.04; additional antibiotic required rate per patient-year 2.3, 95\% CI 2.1-3.4, in the azithromycin group versus placebo 3.6, 95\% CI 2.9-4.3; p=0.004) [76]. Following our final search date, a study involving children/ adolescents and adults with primary ciliary dyskinesia found 6 months of azithromycin (versus placebo) significantly reduced exacerbation rates (rate ratio 0.45 , 95\% CI $0.26-0.78$ ) [53], a similar effect size to this PICO’s main contributing RCT [17].

Justification of recommendation

Although the overall quality of evidence was low, our strong recommendation is from the large effect on exacerbations, the panel's clinical experience, consistency of effect with adult-based RCTs and preventing exacerbations, being one of the key issues for the PAG. The importance and impact of exacerbations on 
children and families were crucial considerations for the strong recommendation. Also, there was relatively minimal possible harms of the intervention. Indeed, the sole study in the evidence table (supplementary material) with low risk of bias for all factors [17] reported (post hoc analyses) antibiotic use for non-pulmonary infections was significantly lower in the azithromycin group versus placebo (incidence rate ratio 0.50 , 95\% CI $0.31-0.81 ; \mathrm{p}=0.005$ ).

Implementation considerations

While an ECG is not necessary before commencing macrolides, a family history of prolonged QT syndrome, arrhythmias and acute cardiac events should be obtained and, when appropriate, an ECG ordered. Also, azithromycin should not be used in children/adolescents with contraindications to macrolides. This includes those with an abnormal ECG, liver function abnormality and azithromycin hypersensitivity.

Adherence $\geqslant 70 \%$ is important for efficacy [17] as well as reducing antibiotic resistance [77]. Adherence $\geqslant 70 \%$ (versus $<70 \%$ ) in the Australian azithromycin group was associated with lower carriage of any pathogen (OR 0.19, 95\% CI 0.07-0.53) and fewer macrolide-resistant pathogens (OR 0.34, 95\% CI 0.140.81) [77].

\section{PICO Question 6}

In children/adolescents with bronchiectasis, should eradication treatment be used (irrespective of symptoms) when there is a new isolate of a potentially pathogenic microorganism (compared to no eradication treatment)?

\section{Recommendation}

- In children/adolescents with bronchiectasis, we suggest eradication therapy following an initial or new detection of $P$. aeruginosa. (Conditional recommendation for the intervention, very low quality of evidence.)

Remarks: Evidence in bronchiectasis is indirect and limited to three small observational studies in adults focused on $P$. aeruginosa eradication. However, we suggest that eradication therapy should commence promptly after confirming $P$. aeruginosa is present (figure 3). Due to lack of evidence, we are unable to comment on eradication treatment for pathogens other than $P$. aeruginosa, which is informed on a case-by-case basis according to the clinical status of the child and the pathogen type. Antibiotic treatment should be made available in every setting where children/adolescents with bronchiectasis are managed.

\section{Summary of evidence}

There were no published studies in children/adolescents. Evidence was from three before-and-after trials in adults [78-80] who underwent eradication for $P$. aeruginosa. These indicate patients may experience improved QoL (compared to pre-eradication) and reduced exacerbation rates and hospitalisation. One study reported the mean number of antibiotic courses was 3.93 in the year before and 2.09 in the year post-eradication $(p=0.002)$ [79]. Another study reported significant reductions in exacerbations, antibiotic use and hospitalisations (mean \pm SD total exacerbations $3.4 \pm 4.21$ in the year before and $1.98 \pm 3.62$ in the year during eradication using inhaled colistin; $\mathrm{p}<0.001)$. Corresponding values for hospitalisation and cycles of antibiotics were $1.94 \pm 2.8$ and $1.18 \pm 1.73$ ( $p=0.018$ ), respectively [80]. The earlier study reported a non-significant reduction in the mean number of hospitalisations (0.39 pre-eradication and 0.29 post-eradication) [79]. However, before-and-after studies are subject to bias, including Hawthorne effects and regression to the mean.

In one study, 11 out of 28 patients who received eradication therapy were without $P$. aeruginosa at 15 months [78]; in another study, 13 out of 24 patients were also without this pathogen at a median 14.3 months [79]. The most recent study [80] reported that eight out of 35 (22.9\%) patients who received 2 weeks of intravenous antibiotics and another five out of 50 (10\%) patients who received 3 weeks of oral anti-pseudomonal treatment had eradicated P. aeruginosa. The 41 out of 67 (61.2\%) who were then treated further with inhaled colistin were free of $P$. aeruginosa 3 months later and $40.3 \%$ were free at 12 months.

Other supportive evidence

Limited supportive data from a recent CF-related systematic review [81] found eradicating $P$. aeruginosa with nebulised antibiotics either alone or combined with oral antibiotics, compared to placebo or no treatment, can eradicate the organism for up to 2 years [81]. However, the impact on clinical outcomes is uncertain. A second CF review [82] on recent detection of methicillin-resistant Staphylococcus aureus in the lower airways reported short-term (28 days) eradication rates are better in those receiving targeted antibiotic treatment, but the effects are not sustained and clinical benefits uncertain. 


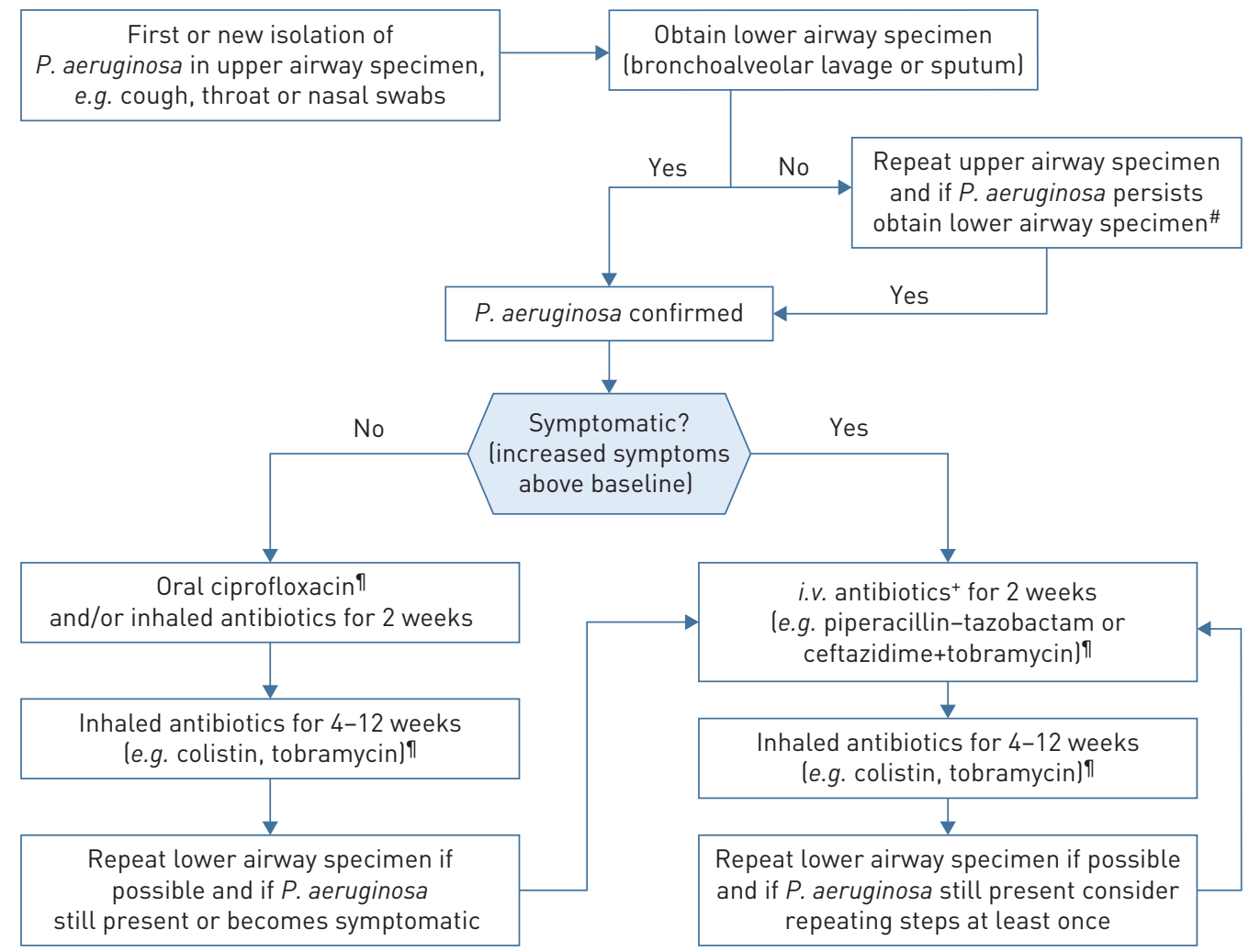

FIGURE 3 Suggested management approach used by the panel when Pseudomonas aeruginosa is first or newly isolated in a child with bronchiectasis. The suggested approach depends upon 1) the specimen type and 2) whether the child is symptomatic. However, panel members acknowledged the approach to initiating eradication treatment is controversial. Some physicians may still feel it is appropriate to initiate eradication therapy based only on a single upper airway specimen, even when symptoms and evidence of benefit in such circumstances are absent. ": if no lower airway specimen available, no treatment if asymptomatic; treat with intravenous anti-pseudomonal antibiotics for 2 weeks if symptomatic. ": antibiotic choices are dependent upon patient factors (e.g. adherence, tolerance and preference), availability of antibiotics and $P$. aeruginosa susceptibility profile. ${ }^{+}$: although there is no trial evidence, many paediatricians use a combination of two intravenous antibiotics. The recommendation for administering two antibiotics when employing short (2-week) intravenous antibiotic courses is made to align with the studies included in the systematic review and the European Respiratory Society adult guidelines [23].

Systematic reviews involving adults with bronchiectasis and chronic $P$. aeruginosa infection reported on eradication using inhaled antibiotics compared to placebo (OR 3.36, 95\% CI 1.63-6.91; p=0.001) with significant reduction in exacerbation frequency (rate ratio $0.81,95 \%$ CI $0.67-0.97 ; \mathrm{p}=0.020$ ) and proportion of patients with one or more exacerbations (OR 0.85, 95\% CI 0.74-0.97; p=0.015) [83]. These data were consistent with another systematic review by different authors that focused on other treatment aspects [84].

Justification of recommendation

There is an established association between lower airway infection with pathogenic microorganisms and deteriorating clinical status and lung function in both the bronchiectasis [85] and CF [86, 87] literature. While there is currently no evidence for early eradication from well-conducted trials in children/adolescents with bronchiectasis, the panel suggests eradication treatment for $P$. aeruginosa. This recommendation places a higher value on the theoretical benefits of eradication and patient/carer values and preferences, and a lower value on possible treatment-related adverse effects.

Implementation considerations

Eradication therapy should employ a targeted antibiotic strategy for the minimum time necessary and measures should be instituted to support full adherence to the prescribed regimen. Like the adult ERS 
guideline [23], without clear evidence for one regimen over another, figure 3 illustrates commonly used approaches in children/adolescents by experts in the field.

\section{Asthma-based medications}

PICO Question 2

In children/adolescents with bronchiectasis, should asthma-type treatments (inhaled corticosteroids (ICS), short-acting $\beta_{2}$-agonists ( $\left.S A B A\right)$ ), long-acting $\beta_{2}$-agonists (LABA)), compared to no asthma-type treatment, be used routinely? Subgroup analyses for a) short- versus long-term and b) stable versus exacerbation states.

\section{Recommendation}

- In children/adolescents with bronchiectasis, we suggest not using ICS with or without LABA routinely in either the short- or long-term, irrespective of stability or exacerbation. (Conditional recommendation, very low quality of evidence.)

Remarks: ICS maybe beneficial in those with eosinophilic airway inflammation. In the absence of any studies on the use with SABA in bronchiectasis, we cannot make any recommendation, but suggest an objective evaluation is undertaken if such asthma-type medications are considered. For some, SABA may be beneficial as pre-airway clearance therapies.

\section{Summary of evidence}

The evidence is based on five RCTs in adults (but not all contributed to all outcomes) and a single observational study in children/adolescents. The latter related to withdrawing ICS and had a high risk of bias, and the reported outcome measures were of doubtful clinical significance $\left(\mathrm{FEV}_{1}\right.$ and provocative concentration causing a $20 \%$ fall in $\mathrm{FEV}_{1}\left(\mathrm{PC}_{20}\right)$ changes were small) [88]. Overall, there is a lack of direct evidence for the use of ICS alone and in combination with LABA in children/adolescents with bronchiectasis. The studies are all very low quality, with only five RCTs in adults identified from systematic reviews [89, 90]. Four of the RCTs involved ICS versus placebo [91-94], while one examined ICS/LABA compared to ICS [95]. Where critical outcomes were obtained from these RCTs, the effect size for benefit was small and non-significant between groups.

For exacerbations, there was no difference between those who received versus those who did not receive ICS (studies $\leqslant 6$ months: average number of participants mean difference -0.17 , 95\% CI $-0.56-0.22$; number of participants with at least one exacerbation OR 0.27 , 95\% CI 0.02-3.09, hospitalisation OR 0.20, 95\% CI 0.02-1.90; studies >6 months: number of participants with improved frequency of exacerbation OR 1.61, 96\% CI 0.68-3.81). See evidence table in the supplementary material for other outcomes.

RCT data in adults with bronchiectasis show increased adverse events when ICS are used and the risk increases with higher ICS doses. Also, there is observational study evidence of increased risk of NTM infection and pneumonia in adults with bronchiectasis and other chronic respiratory diseases who received ICS [96, 97].

\section{Other supportive evidence}

The panel considered that there is good evidence from the non-bronchiectasis literature that ICS can lead to adrenal suppression [98] and growth failure [99-101], as well as other adverse effects [97]. As there is no reason to suppose this would be different in bronchiectasis, these medications should not be used routinely unless there is objective evidence of benefit. Further, in adults with bronchiectasis, those commencing ICS had poorer outcomes than those starting macrolides (higher risk of hospitalised respiratory infection (HR 1.39, 95\% CI 1.23-1.57) and exacerbations (HR 1.56, 95\% CI 1.49-1.64)) [102].

\section{Justification of recommendation}

The evidence (albeit very low quality) shows a lack of efficacy for these medications. The panel considered the overall weight of the literature, examining the efficacy and safety of ICS in adults and in other conditions. This, combined with biological plausibility and the absence of any reason to suppose the effects are any different in children/adolescents, would lead most clinicians to be very concerned about potential adverse events from ICS, alone and in combination with LABA. Data on important adverse events are supported by systematic reviews in other chronic respiratory diseases. These potential serious adverse events (increased risk of NTM infection, pneumonia and tuberculosis), with strong biological plausibility for causation, suggest against routine use of ICS with or without LABA in either the short- or long-term. Also, there is supportive evidence of other possible harm as outlined above. 
The fiscal cost associated with ICS prescription globally is substantial. Hence, prescribing ICS/LABA needs positive justification, which cannot be found in the current literature.

Implementation considerations

As bronchiectasis and eosinophilic asthma symptoms overlap, we recommend that if treatment with ICS or ICS/LABA is contemplated, every effort should be made to document acute bronchodilator sensitivity (acute spirometric response to SABA), atopy (skin prick tests and specific IgE) and airway eosinophilia (peripheral blood eosinophil count, sputum eosinophils and exhaled nitric oxide). It should be noted that the sensitivity and specificity of all these tests vary across the globe, but if there is no evidence of atopy or airway eosinophilia in a given patient, ICS and ICS/LABA are unlikely to have a role. If a blind trial of ICS or ICS/LABA is contemplated, because the above tests are equivocal or unavailable, objective evidence of benefit should be obtained if the medications are continued.

There is, however, a subgroup with asthma-type responses where using SABA pre-ACT may prove useful.

\section{Surgery}

Narrative Question 7

In children/adolescents with bronchiectasis, what factors should be taken into account when considering surgical removal of the diseased lung?

Usual practice statement

It is important to emphasise that surgery is rarely undertaken in the panel's experience, although we are aware that it is not uncommon in some settings. Surgery is only considered after maximal medical therapies (e.g. ACT, long-term antibiotics, etc.) have failed and the child/adolescent's QoL remains significantly impaired. When contemplated, a multidisciplinary approach is essential, and the decision should be based on the individual's clinical state and local surgical expertise.

\section{Recommendation}

- In children/adolescents with bronchiectasis, we recommend when considering surgery, factors to be taken into account include age, symptoms and disease burden, localisation of the bronchiectatic areas on chest CT scans, the underlying aetiology (influencing recurrence of disease), facility where surgery is undertaken (surgical expertise and availability of pre- and post-surgical care), and optimisation of the child's clinical state. (Strong recommendation, very low quality of evidence stemming from the narrative review.)

Remarks: The benefits from surgery are higher in those with localised disease where complete resection can be done and when the disease is not recurrent (i.e. absence of underlying aetiology such as immunodeficiency). Careful pre-operative evaluation as well as rehabilitation post-surgery improves outcome. Ideally, bronchoscopy and bronchoalveolar lavage are performed prior to surgery to exclude a foreign body and obtain microbiological samples. A ventilation/perfusion scan to delineate non-ventilated areas confirming the localised disease to plan for the surgery is likely beneficial. Optimisation of the child/ adolescent's clinical state, including using appropriately targeted antibiotics, ACT and improving nutritional status, pre- and post-surgery is also necessary.

\section{Summary of evidence}

The narrative summary only identified observational studies. There was a single prospective [103] study and the rest $(n=43)$ were retrospective. One meta-analysis [104] included the results of five paediatric studies. Also, 18 out of 42 (43\%) studies were undertaken by surgical groups from one country, thus raising the possibility of selection and reporting bias. The limited evidence suggests better results if surgery is undertaken in specialised centres after a series of tests (ventilation/perfusion scan, bronchoscopy and chest CT scans) and optimising the patient's lung function pre-surgery. Factors to be considered include the underlying aetiology (influencing recurrence of disease), location and extent of disease (lobes affected).

Other supportive evidence

Surgery for bronchiectasis is now undertaken rarely in high-income countries, but is not uncommon in low- to middle-income countries. Members of the panel rarely advocate surgery to control bronchiectasis.

\section{Justification of recommendation}

Although evidence for assessing factors favouring lung surgery for children/adolescents with bronchiectasis is very low, the data from the studies are consistent and inform the current standard of care 
in specialist settings. Also, the panel and PAG expressed the view that standardised clinical care is very important when surgery is being considered, allowing risks versus benefits to be balanced.

Implementation considerations

Increasing accessibility to a multidisciplinary team with expertise in optimal pre-operative evaluation and careful patient selection is recommended. Video-assisted thoracoscopic surgery, compared to open thoracotomy, is associated with fewer complications and shorter post-operative hospital stay.

\section{Systematic care}

Narrative Question 3

In children/adolescents with bronchiectasis, should attention be paid to other paediatric systematic care issues (nutrition, aerobic and non-aerobic exercise, psychological support, equipment care, vaccinations, etc.)?

\section{Recommendations}

- In children/adolescents with bronchiectasis, we suggest that nutrition is optimised, including vitamin D status. (Conditional recommendation, very low quality of evidence stemming from the narrative review.)

Remark: There is no evidence upon which to recommend additional nutritional supplements.

- In children/adolescents with bronchiectasis, we suggest that exercise is encouraged on an ongoing basis; short periods of exercise training are unlikely to have a long-term effect. (Conditional recommendation, very low quality of evidence stemming from the narrative review.)

Remark: There is insufficient evidence to make a recommendation for establishing formal exercise and rehabilitation programmes.

- In children/adolescents with bronchiectasis, we suggest they are fully immunised according to their national immunisation programmes, including pneumococcal and annual seasonal influenza vaccines if these are not part of this programme. (Conditional recommendation, very low quality of evidence stemming from the narrative review.)

- In children/adolescents with bronchiectasis, we suggest they receive psychological support and education on equipment use and care. (Conditional recommendation, very low quality of evidence stemming from the narrative review.)

\section{Summary of evidence}

The evidence is overall of very low quality. The 14 included studies were: nine reviews, two RCTs [105, $106]$ in adults, one RCT in children/adolescents [107] and two observational studies [108, 109].

The desirable effects of routine immunisation, exercise and good nutrition are undeniable, but their magnitude is uncertain. Additional vaccinations for children/adolescents with bronchiectasis are likely beneficial, but the quality of the evidence is very low $[107,110]$. The positive effects of psychological support and teaching appropriate equipment use and care for children/adolescents with chronic illness are also likely highly desirable, but there are no data on type, duration or intensity of support or how to assist with maintaining equipment. The data relevant for vitamin D were limited to adult-based studies [111].

Exercise training for short periods is unlikely to have prolonged effects and the implication is that exercise support must be ongoing. There is low quality of evidence supporting fewer pulmonary exacerbations and longer time-to-first exacerbation with exercise training. There are no agreed formal pulmonary rehabilitation programmes in children/adolescents and there are no data on what exercise interventions are most important. Whether a formal exercise programme is superior to encouragement of an active lifestyle is unclear.

Justification of recommendation

Recommendations are based upon placing a higher value on low/moderate quality of evidence for clinical improvement over a low value for concerns over uncertainty of magnitude and duration of benefit. The need for good nutrition, immunisation and exercise in childhood would be widely supported.

Implementation considerations

Increase the accessibility of children/adolescents to centres practising standard of care in low- and middle-income countries.

Narrative Question 4c

How should cross-infection be minimised? 
Recommendation

- In children/adolescents with bronchiectasis, we suggest that they and their family are counselled on cough and hand hygiene. Wherever possible, they should also avoid those with symptoms of viral respiratory infections. Children/adolescents managed within a CF clinic must follow their infection control policies. (Conditional recommendation, very low quality of evidence stemming from the narrative review.)

Addendum: The guideline was written pre-COVID-19, but in view of this, children/adolescents with bronchiectasis should follow measures recommended by local health authorities.

Monitoring

Narrative Question $4 b$

How frequently should patients be seen in outpatient clinics?

\section{Recommendation}

- In children/adolescents with bronchiectasis, we suggest they are reviewed every 3-6 months in outpatient clinics to monitor their general wellbeing, respiratory status, including lung function when age-appropriate, and to detect any complications. (Conditional recommendation, very low quality of evidence stemming from the narrative review.)

\section{Narrative Question $4 a$}

How often should airway microbiology testing be conducted in outpatients?

\section{Recommendation}

- In children/adolescents with bronchiectasis, we suggest in those able to expectorate that routine spontaneous or induced sputum samples are collected every 6-12 months as a means of identifying new pathogens, specifically $P$. aeruginosa, and to help guide initial empiric antibiotic therapy for future exacerbations. (Conditional recommendation, very low quality of evidence stemming from the narrative review.)

Summary of evidence (for NQ4a-c)

The data presented in the supplementary material support outpatient clinic reviews every 3-6 months and standard infection control policies without segregating patients. However, for each of the three parts of NQ4, there are no RCTs and evidence is based predominantly on observational studies in both children/ adolescents [14, 35, 51, 112-115]. The desirable frequency of outpatient clinic attendance and airway microbiology surveillance is dependent upon patient factors (e.g. age, underlying aetiology, illness severity, comorbidities and ability to reliably expectorate spontaneous or induced sputum) and circumstances (e.g. travelling long distances for clinic attendance). Outpatient sputum culture surveillance every 6-12 months is based on expert opinion [24].

Limited evidence prevents robust recommendations on infection control policies for patients with bronchiectasis. If managed within a CF centre, local CF infection control policies should be followed and direct contact with CF patients avoided. Standard infection control procedures should be discussed with patients/families, and hand and cough hygiene measures followed. Influenza and other recommended vaccines by national authorities are also endorsed.

Post-writing the guidelines, the onset of the COVID-19 pandemic led local health authorities to introduce additional non-pharmacological public health measures to interrupt virus transmission.

Other supportive evidence

The panel's collective clinical experience supports the approach outlined in the research evidence and the pre-COVID-19 pragmatic recommendations of the European Bronchiectasis Network (EMBARC) statement on infection control [116].

\section{Justification of recommendation}

Although the quality of the evidence for the above interventions leading to improving clinical outcomes is very low, the suggestions above were based upon indirect evidence that current standard of care in specialist settings leads to improved lung function post-diagnosis. Also, the panel and PAG advocated for regular clinical care and monitoring by specialists, and for advice on avoiding cross-infection. 
Implementation considerations

Increased accessibility of children/adolescents to centres practising standards of care. It is also important to educate clinicians, families and patients on the role of surveillance sputum cultures in those with clinically stable bronchiectasis. As upper airway swabs are unreliable at predicting lower airway pathogens, spontaneous or induced sputum samples in children/adolescents able to expectorate are recommended for surveillance cultures. Bronchoalveolar lavage is reserved for treatment failures, especially if sputum cultures are negative and/or unusual pathogens are suspected.

\section{Narrative Question 5d}

Are there any routine tests that should be undertaken to detect complications when attending outpatient clinics?

\section{Recommendation}

- In children/adolescents with bronchiectasis, we suggest the following routine tests are undertaken to detect complications when attending outpatient clinics: 1) lung function (spirometry for $\mathrm{FEV}_{1}$ and FVC) when age-appropriate, 2) sputum when they can expectorate and 3) pulse oximetry. (Conditional recommendation, very low quality of evidence stemming from the narrative review.)

\section{Narrative Question $5 f$}

In gradually deteriorating (i.e. non-acute) patients, what investigations should be undertaken?

\section{Recommendation}

- In children/adolescents with bronchiectasis whose clinical status is gradually deteriorating, we suggest they are assessed for new infections (sputum or lower airway microbiology) and possible comorbidities (e.g. asthma, gastro-oesophageal reflux disease, nutritional deficiencies, dental or sleep disorders, etc.). (Conditional recommendation, very low quality of evidence stemming from the narrative review.)

Remarks: These children/adolescents often require hospitalisation for intravenous antibiotics and airway clearance therapy.

Narrative Question 5e

When should repeat chest CT scans be undertaken?

\section{Recommendation}

- In children/adolescents with bronchiectasis, we suggest the decision to repeat chest CT scans is individualised based on the clinical status and setting. (Conditional recommendation, very low quality of evidence stemming from the narrative review.)

Remarks: Repeat chest CT scans should be considered to answer a question which will change management.

\section{Summary of evidence (for NQ5d-f)}

The evidence provided in the narrative summary found only indirect evidence for using routine tests to detect complications of bronchiectasis, investigations required for gradually deteriorating patients and whether chest CT scans should be repeated. Our search did not identify any RCTs that address these questions. The current evidence is based on 11 observational studies (10 retrospective and one prospective cross-sectional study [41]) and it is highly unlikely that any RCT will be undertaken.

The desirable interventions are patient-specific (e.g. age (young children require general anaesthesia for chest CT scans), illness severity and costs of tests) and circumstance-specific (e.g. underlying disease and patients travelling long distances). Thus, desirable effects vary.

Specialists in respiratory clinics at tertiary paediatric hospitals currently use a model of care that, although not fully described, includes standardised care assessing stability and detecting deterioration based on clinical history and investigations. In these settings, studies report this model leads to improved lung function post-diagnosis of bronchiectasis [2, 14, 35]. The monitoring component of the standardised care includes 3-6-monthly clinical reviews with: 1) spirometry to assess FEV 1 and FVC, and 2) assessment for new infection (sputum for bacteria culture during exacerbations and 6 monthly as routine) and assessing (and when indicated investigating) for new comorbidities (e.g. asthma, gastro-oesophageal reflux disease, nutritional deficiencies, dental or sleep disorders, etc.). 
Tertiary paediatric respiratory clinics monitor clinical symptoms, frequency and severity of respiratory exacerbations, and lung function indices. When deterioration occurs, the narrative evidence [2, 14, 35, 117, 118] supports assessing and investigating for the treatable traits listed above.

Evidence from the narrative summary found several studies repeating chest CT scans in children/ adolescents with bronchiectasis [41, 118-122]. However, the reasons given for repeating scans were largely based on clinical grounds. Indications include documenting reversal of bronchiectasis (e.g. for medical insurance or reducing care burden for parents) or when there is an acute or gradual deterioration (e.g. to assess for new treatable disease or justify more intensive treatments).

Other supportive evidence

Other supportive data include reducing exacerbations by following standards of care [123]. The panel's collective clinical experience supports the approach outlined in the supplementary material for NQ5.

Justification of recommendation

Although the evidence for the above interventions improving clinical outcomes is very low, the suggestions above were based upon indirect evidence that the current standard of care in specialist settings leads to improved lung function post-diagnosis. Also, the panel and PAG advocated for standardised clinical care, especially in primary care settings.

\section{Implementation considerations}

Increase accessibility of children/adolescents to centres practising the recommended standard of care. Obtaining additional CT scans needs to be balanced against the reported increased life-time cancer risk, which is age- and dose-dependent. Although relatively negligible at the individual level and lower with newer CT protocols, children previously have been estimated to have 10 times increased life-time cancer risk following CT scans compared to middle-aged adults undergoing this investigation [124]. Currently, specialists in tertiary paediatric respiratory clinics individualise the need to repeat the chest CT scans.

Reversibility and prevention

Narrative Question 2

In children/adolescents, is bronchiectasis a) reversible and/or b) preventable?

In some children/adolescents, their bronchiectasis is reversible and/or preventable. Factors important for reversibility and/or prevention of bronchiectasis include early identification and treatment of inhaled foreign bodies, preventing early and severe pneumonia, preventing recurrent protracted bacterial bronchitis, treating primary immunodeficiency disorders causing bronchiectasis, promoting breastfeeding and immunisation, and avoiding tobacco smoke and other pollutants.

\section{Good practice statement}

- In children/adolescents with bronchiectasis, we suggest wherever possible, interventions that reverse and/or prevent bronchiectasis are undertaken. However, these measures are context- and patient-specific.

\section{Summary of evidence}

While the evidence is very low, all six studies showed that with appropriate management early bronchiectasis in some children/adolescents is reversible and thus preventable [2, 37, 118, 125-127]. The resolution or improvement rates after appropriate treatment in children/adolescents with radiographically confirmed bronchiectasis may be as great as 64\% [2]. However, the proportion of resolution or improvement likely varies with bronchiectasis severity, underlying aetiology, treatment provided and how bronchiectasis was defined (the diagnostic criteria used).

Identifying and removing aspirated foreign bodies from the airways, especially within 14 days, prevents bronchiectasis developing [128]. When treatment is delayed $>30$ days, bronchiectasis occurred in $60 \%$ of children with retained foreign bodies [129]. Treating primary immunodeficiency is warranted, irrespective of whether bronchiectasis is present.

A single-blind RCT showed community clinic-based primary care review of young children in New Zealand post-hospitalisation for pneumonia/bronchiolitis did not prevent future bronchiectasis (found in $3.7 \%$ of the cohort) [130]; thus, interventions other than clinical review are required. There is only indirect observational evidence on other potential risk factors for developing bronchiectasis in children/adolescents, 
TABLE 2 Summary of PICO questions and recommendations

$\begin{array}{lll}\text { PICO Title Recommendations } & \end{array}$

1 In children/adolescents suspected of bronchiectasis: a) Should multidetector chest computed tomography (MDCT) scans with high-resolution CT (HRCT) be used instead of conventional HRCT alone for diagnosing bronchiectasis? b) What CT criteria for broncho-arterial dilatation (BAR) should be used?

In children/adolescents with bronchiectasis, should asthma-type treatments (inhaled corticosteroids (ICS), short-acting $\beta_{2}$-agonists (SABA), long-acting $\beta_{2}$-agonists (LABA)), compared to no asthma-type treatment, be used routinely? Subgroup analyses for a) short- versus long-term and b) stable versus exacerbation states.

3 In children/adolescents with bronchiectasis, should mucoactive agents (compared to no mucoactive agents) be used routinely? Subgroup analyses for a) short- versus long-term, b) stable versus exacerbation states and c) type of mucoactive agent.

$4 \quad$ In children/adolescents with bronchiectasis, should regular airway clearance techniques (ACT) (compared to no ACT) be undertaken? Subgroup analyses for a) short- versus long-term and b) stable versus exacerbation states.

In children/adolescents with bronchiectasis, should systemic courses of antibiotics (compared to no antibiotics) be used to treat an acute respiratory exacerbation (type and duration)?
- In children/adolescents suspected of bronchiectasis, we suggest that high-resolution MDCT scans with HRCT be used instead of conventional HRCT to diagnose bronchiectasis in children/adolescents. (Conditional recommendation, very low quality of evidence.)

- In children/adolescents suspected of bronchiectasis, we suggest that paediatric-derived BAR (defined by the ratio of the inner diameter of the airway to the outer diameter of the adjacent artery) of $>0.8$ is used to define abnormality instead of the adult cut-off of $>1-1.5$. (Conditional recommendation, very low quality of evidence stemming from the narrative review.)

- In children/adolescents with bronchiectasis, we suggest not using ICS with or without LABA routinely in either the short- or long-term, irrespective of stability or exacerbation. (Conditional recommendation, very low quality of evidence.)

Remarks: ICS maybe beneficial in those with eosinophilic airway inflammation. In the absence of any studies on the use with SABA in bronchiectasis, we cannot make any recommendation, but suggest an objective evaluation is undertaken if such asthma-type medications are considered. For some, SABA may be beneficial as pre-airway clearance therapies.

- In children/adolescents with bronchiectasis, we recommend that recombinant human DNase (rhDNase) is not used routinely. (Strong recommendation, very low quality of evidence.)

- In children/adolescents with bronchiectasis, we suggest that bromhexine is not used routinely. (Conditional recommendation, very low quality of evidence.)

- In children/adolescents with bronchiectasis, we suggest that neither inhaled mannitol nor hypertonic saline are used routinely. (Conditional recommendation, very low quality of evidence.)

Remarks: Inhaled mannitol or $6-7 \%$ hypertonic saline may be considered in selected patients e.g. those with high daily symptoms, frequent exacerbations, difficulty in expectoration and/or poor QoL. If well tolerated, the use of hypertonic saline or mannitol could improve the QoL and facilitate expectoration. For hypertonic saline and mannitol, children should be old enough to tolerate these interventions, and the panel also considered that short-acting $\beta_{2}$-agonists should be used prior to inhaling either hypertonic saline or mannitol. The first dose of hypertonic saline or mannitol should be administered under medical supervision. The substantially higher cost of mannitol compared with hypertonic saline should also be taken into consideration.

- In children/adolescents with bronchiectasis, we recommend that they are taught and receive regular ACT or manoeuvres. (Strong recommendation, low quality of evidence.)

Remarks: Individualised ACT that are development- and age-appropriate are best taught by a paediatric-trained chest physiotherapist (figure 2). The frequency of ACT is best individualised. As children/adolescents mature, techniques may need to be changed, and thus the ACT type and frequency is best reviewed at least biannually by physiotherapists with expertise in paediatric respiratory care. During acute exacerbations of bronchiectasis, children/adolescents should receive ACT more frequently.

- In children/adolescents with bronchiectasis and an acute respiratory exacerbation, we recommend a systemic course of an appropriate antibiotic is used for 14 days. (Strong recommendation, moderate quality of evidence.) Remarks: The empiric antibiotic of choice is amoxicillin-clavulanate, but the type of antibiotics chosen should be based on the patient's airway cultures (e.g. those with Pseudomonas aeruginosa require different treatment regimens to those without) and history of antibiotic hypersensitivity reactions. When the exacerbation is severe (e.g. the child/adolescent is hypoxic) and/or when the child/adolescent does not respond to oral antibiotics, intravenous antibiotics will be needed. 


\section{TABLE 2 Continued}

$\begin{array}{lll}\text { PICO Title Recommendations } & \end{array}$

6 In children/adolescents with bronchiectasis, should eradication treatment be used (irrespective of symptoms) when there is a new isolate of a potentially pathogenic microorganism (compared to no eradication treatment)?
In children/adolescents with bronchiectasis and recurrent exacerbations, should long-term ( $\geqslant 2$ months) macrolide antibiotics (compared to no antibiotics) be used to reduce exacerbations?
- In children/adolescents with bronchiectasis, we suggest eradication therapy following an initial or new detection of $P$. aeruginosa. (Conditional recommendation for the intervention, very low quality of evidence.) Remarks: Evidence in bronchiectasis is indirect and limited to three small observational studies in adults focused on $P$. aeruginosa eradication. However, we suggest that eradication therapy should commence promptly after confirming $P$. aeruginosa is present (figure 3). Due to lack of evidence, we are unable to comment on eradication treatment for pathogens other than $P$. aeruginosa, which is informed on a case-by-case basis according to the clinical status of the child and the pathogen type. Antibiotic treatment should be made available in every setting where children/adolescents with bronchiectasis are managed.

- In children/adolescents with bronchiectasis and recurrent exacerbations, we recommend treatment with long-term macrolide antibiotics to reduce exacerbations. (Strong recommendation, low quality of evidence.) Remarks: Based on the panel's experience, we suggest long-term macrolide antibiotics only in those who have had more than one hospitalised or three or more non-hospitalised exacerbations in the previous 12 months. Such a course should be for at least 6 months with regular reassessment to determine whether the antibiotic continues to provide a clinical benefit. Children/ adolescents receiving longer treatment courses (>24 months) should continue to be evaluated for risk versus benefit. This suggestion is in the context of lacking data concerning when long-term azithromycin should be initiated, and the need for caution because of increasing antibiotic resistance among bacterial pathogens within patients and the community. While NTM are very rarely detected in children/adolescents with bronchiectasis, we suggest a lower airway specimen is obtained (when possible) to exclude their presence before commencing long-term macrolide antibiotics. We encourage strategies to ensure adherence to the macrolide regimen as $\geqslant 70 \%$ adherence improves efficacy and reduces antibiotic resistance.

QoL: quality of life; NTM: non-tuberculous mycobacteria.

which includes strategies targeting household crowding, prematurity, and frequent, early-onset and severe acute lower respiratory tract infections (especially hospitalised pneumonia) [131, 132]. Preventing recurrent protracted bacterial bronchitis, non-typeable Haemophilus influenzae lower airway infection and increasing breastfeeding may also prevent future bronchiectasis [131, 133]. However, the evidence is low and the effect size is uncertain.

\section{Justification of recommendation}

The evidence for preventing and/or reversing bronchiectasis in children/adolescents is very low to low. We called this a best practice statement, as not seeking to prevent or reverse a disease (if possible) would be unethical.

\section{Implementation considerations}

Access and strategies to improve early diagnosis and interventions to prevent and/or reverse bronchiectasis are required.

\section{Conclusions}

The recommendations from the ERS Task Force on the management of children and adolescents with bronchiectasis are summarised in tables 2 and 3. The guidelines aim to assist health professionals with optimising postnatal lung growth, preserving lung function, enhancing QoL, minimising exacerbations, preventing complications and, if possible when diagnosed early, reversing bronchial wall dilatation as a marker of structural lung injury. In so doing, we also seek to balance benefits and risks associated with each of the recommended treatment approaches. As knowledge gaps and identified research priorities are addressed, the guideline will need ongoing development in the years ahead. 
TABLE 3 Summary of narrative questions (NQS) and recommendations

\section{NQ Title}

1 In children/adolescents with suspected or confirmed bronchiectasis, what standard tests that impact on clinical outcomes should be undertaken when managing this group of patients?

In children/adolescents, is bronchiectasis a) reversible and/or b) preventable?

In children/adolescents with bronchiectasis, should attention be paid to other paediatric systematic care issues (nutrition, aerobic and non-aerobic exercise, psychological support, equipment care, vaccinations, etc.)?

When monitoring children/adolescents with bronchiectasis: a) How often should airway microbiology testing be conducted in outpatients? b) How frequently should patients be seen in outpatient clinics? c) How should cross-infection be minimised?

\section{Recommendations}

- In children/adolescents with suspected or confirmed bronchiectasis, we suggest they have a minimum panel of tests undertaken, as done currently by most experts in the field. (The minimum panel of tests comprises: 1 ) chest CT scan (to diagnose bronchiectasis), 2) sweat test, 3) lung function tests (in children/adolescents who can perform spirometry), 4) full blood count, 5) immunological tests (total IgG, IgA, IgM, IgE and specific antibodies to vaccine antigens) and 6) lower airway bacteriology.) (Conditional recommendation, very low quality of evidence stemming from the narrative review.)

- In selected children/adolescents with bronchiectasis, we suggest additional tests are considered based on their clinical presentation. These include additional in-depth immunological assessments (in consultation with a paediatric immunologist), diagnostic bronchoscopy with bronchoalveolar lavage analysis (microbiology), and tests for airway aspiration, primary ciliary dyskinesia and gastro-oesophageal disease. (Conditional recommendation, low quality of evidence stemming from the narrative review.)

Remarks: In settings where tuberculosis or HIV have a high prevalence and/or there is a history of close contact with tuberculosis, assessment for tuberculosis infection/disease or HIV, respectively, is also undertaken as part of the minimum panel of tests.

In some children/adolescents, their bronchiectasis is reversible and/or preventable. Factors important for reversibility and/or prevention of bronchiectasis include early identification and treatment of inhaled foreign bodies, preventing early and severe pneumonia, preventing recurrent protracted bacterial bronchitis, treating primary immunodeficiency disorders causing bronchiectasis, promoting breastfeeding and immunisation, and avoiding tobacco smoke and other pollutants.

Good practice statement:

- In children/adolescents with bronchiectasis, we suggest wherever possible, interventions that reverse and/or prevent bronchiectasis are undertaken. However, these measures are context- and patient-specific.

- In children/adolescents with bronchiectasis, we suggest that nutrition is optimised, including vitamin D status. (Conditional recommendation, very low quality of evidence stemming from the narrative review.)

Remarks: There is no evidence upon which to recommend additional nutritional supplements.

- In children/adolescents with bronchiectasis, we suggest that exercise is encouraged on an ongoing basis; short periods of exercise training are unlikely to have a long-term effect (Conditional recommendation, very low quality of evidence stemming from the narrative review.)

Remarks: There is insufficient evidence to make a recommendation for establishing

formal exercise and rehabilitation programmes.

- In children/adolescents with bronchiectasis, we suggest that they are fully immunised according to their national immunisation programmes, including pneumococcal and seasonal influenza vaccines if these are not part of this programme. (Conditional recommendation, very low quality of evidence stemming from the narrative review.)

- In children/adolescents with bronchiectasis, we suggest that they receive psychological support and education on equipment use and care (Conditional recommendation, very low quality of evidence stemming from the narrative review.)

- In children/adolescents with bronchiectasis, we suggest in those able to expectorate that routine spontaneous or induced sputum samples are collected every 6-12 months as a means of identifying new pathogens, specifically $P$. aeruginosa, and to help guide initial empiric antibiotic therapy for future exacerbations. (Conditional recommendation, very low quality of evidence stemming from the narrative review.)

- In children/adolescents with bronchiectasis, we suggest they are reviewed every 3-6 months in outpatient clinics to monitor their general wellbeing, respiratory status, including lung function when age-appropriate, and to detect any complications. (Conditional recommendation, very low quality of evidence stemming from the narrative review.) 
TABLE 3 Continued

NQ Title bronchiectasis: d) Are there any routine tests that should be undertaken to detect complications when attending outpatient clinics? e) When should repeat chest CT scans be undertaken? f) In gradually deteriorating (i.e. non-acute) patients, what investigations should be undertaken?

In children/adolescents with bronchiectasis, what criteria should be used to define an exacerbation?

In children/adolescents with bronchiectasis, what factors should be taken into account when considering surgical removal of the diseased lung?

\section{Recommendations}

- In children/adolescents with bronchiectasis, we suggest that they and their family are counselled on cough and hand hygiene. Wherever possible, they should also avoid those with symptoms of viral respiratory infections. Children/ adolescents managed within a CF clinic must follow their infection control policies. (Conditional recommendation, very low quality of evidence stemming from the narrative review.)

Addendum: The guideline was written pre-COVID-19, but in view of this, children/ adolescents with bronchiectasis should follow measures recommended by local health authorities.

- In children/adolescents with bronchiectasis, we suggest the following routine tests are undertaken to detect complications when attending outpatient clinics: 1) lung function (spirometry for $\mathrm{FEV}_{1}$ and FVC) when age-appropriate, 2) sputum when they can expectorate and 3) pulse oximetry. (Conditional recommendation, very low quality of evidence stemming from the narrative review.)

- In children/adolescents with bronchiectasis, we suggest the decision to repeat chest CT scans is individualised based on the clinical status and setting. (Conditional recommendation, very low quality of evidence stemming from the narrative review.)

Remarks: Repeat chest CT scans should be considered to answer a question which will change management.

- In children/adolescents with bronchiectasis whose clinical status is gradually deteriorating, we suggest they are assessed for new infections (sputum or lower airway microbiology) and possible comorbidities (e.g. asthma, gastro-oesophageal reflux disease, nutritional deficiencies, dental or sleep disorders, etc.). (Conditional recommendation, very low quality of evidence stemming from the narrative review.)

Remarks: These children/adolescents often require hospitalisation for intravenous antibiotics and airway clearance therapy.

For clinical purposes:

- In children/adolescents with bronchiectasis, we suggest that a respiratory exacerbation is considered present when a child/adolescent has increased respiratory symptoms (predominantly increased cough with or without increased sputum quantity and/or purulence) for $\geqslant 3$ days. (Conditional recommendation, low quality of evidence stemming from the narrative review.) Remarks: Other important, but less common, respiratory symptoms such as haemoptysis, chest pain, breathlessness and wheeze may not be present. Clinicians should not rely on changes in chest auscultation findings and chest radiographs to diagnose an exacerbation as, although important, these findings are not always present. Systemic symptoms (fever, fatigue, malaise, change in child's behaviour and appetite) may also herald onset of an exacerbation, but are non-specific. Blood markers (e.g. elevated C-reactive protein, neutrophilia and interleukin-6) provide supportive evidence of the presence of an exacerbation. However, these indices are less important in defining exacerbations, but are likely useful for research purposes. Also, markers like interleukin- 6 are not standard clinical tests.

- In children/adolescents with bronchiectasis, we recommend that the presence of dyspnoea (increased work of breathing) and/or hypoxia should be considered a severe exacerbation, irrespective of duration. (Strong recommendation, low quality of evidence stemming from the narrative review.) Usual practice statement: It is important to emphasise that surgery is rarely undertaken in the panel's experience, although we are aware that it is not uncommon in some settings. Surgery is only considered after maximal medical therapies (e.g. ACT, long-term antibiotics, etc.) have failed and the child/ adolescent's QoL remains significantly impaired. When contemplated, a multidisciplinary approach is essential, and the decision should be based on the individual's clinical state and local surgical expertise.

- In children/adolescents with bronchiectasis, we recommend when considering surgery, factors to be taken into account include age, symptoms and disease burden, localisation of the bronchiectatic areas on chest CT, the underlying aetiology (influencing recurrence of disease), facility where surgery is undertaken (surgical expertise and availability of pre- and post-surgical care), 
and optimisation of the child's clinical state. (Strong recommendation, very low quality of evidence stemming from the narrative review.)

Remarks: The benefits from surgery are higher in those with localised disease where complete resection can be done and when the disease is not recurrent (i.e. absence of underlying aetiology such as immunodeficiency). Careful pre-operative evaluation as well as rehabilitation post-surgery improves outcome. Ideally, bronchoscopy and bronchoalveolar lavage are performed prior to surgery to exclude a foreign body and obtain microbiological samples. A ventilation/perfusion scan to delineate non-ventilated areas confirming the localised disease to plan for the surgery is likely beneficial. Optimisation of the child/adolescent's clinical state, including using appropriately targeted antibiotics, $\mathrm{ACT}$ and improving nutritional status, pre- and post-surgery is also necessary.

$\mathrm{CT}$ : computed tomography; $\mathrm{CF}$ : cystic fibrosis; $\mathrm{FEV}_{1}$ : forced expiratory volume in $1 \mathrm{~s}$; FVC: forced vital capacity; ACT: airway clearance techniques; QoL: quality of life.

Acknowledgements: We thank Elizabeth Stovold (Information Specialist from the Cochrane Airways Group) for having undertaken all the searches and Rebecca Morgan (McMaster University, Hamilton, ON, Canada) for her initial help in the methodological aspects of this guideline. We are also grateful to the extended ELF's PAG who helped shape the document through framing the PICOs and NQs and selection of outcomes for the PICOs.

The guidelines published by the European Respiratory Society (ERS) incorporate data obtained from a comprehensive and systematic literature review of the most recent studies available at the time. Health professionals are encouraged to take the guidelines into account in their clinical practice. However, the recommendations issued by this guideline may not be appropriate for use in all situations. It is the individual responsibility of health professionals to consult other sources of relevant information, to make appropriate and accurate decisions in consideration of each patient's health condition and in consultation with that patient and the patient's caregiver where appropriate and/or necessary, and to verify rules and regulations applicable to drugs and devices at the time of prescription.

This document was endorsed by the ERS Executive Committee on 15 January 2021.

Conflict of interest: A.B. Chang reports grants from the National Health and Medical Research Council, Australia; other fees to the institution from work relating to being an IDMC Member of an unlicensed vaccine (GlaxoSmithKline) and an advisory member of a study design for an unlicensed molecule for chronic cough (Merck) outside the submitted work. R. Fortescue has nothing to disclose. K. Grimwood reports grants from the National Health and Medical Research Council, Australia, during the conduct of the study. E. Alexopoulou has nothing to disclose. L. Bell has nothing to disclose. J. Boyd is an employee of the European Lung Foundation. A. Bush has nothing to disclose. J.D. Chalmers reports grants and personal fees from AstraZeneca, Boehringer Ingelheim, GlaxoSmithKline and Insmed, personal fees from Chiesi, Novartis and Zambon, grants from Gilead Sciences, outside the submitted work. A.T. Hill has nothing to disclose. B. Karadag has nothing to disclose. F. Midulla has nothing to disclose. G.B. McCallum has nothing to disclose. Z. Powell has nothing to disclose. D. Snijders has nothing to disclose. W-J. Song has nothing to disclose. T. Tonia acts as a methodologist for the European Respiratory Society. C. Wilson has nothing to disclose. A. Zacharasiewicz has nothing to disclose. A. Kantar has nothing to disclose.

Support statement: This work was supported by the European Respiratory Society and the National Health and Medical Research Council, Australia. Funding information for this article has been deposited with the Crossref Funder Registry.

\section{References}

1 Chang AB, Bush A, Grimwood K. Bronchiectasis in children: diagnosis and treatment. Lancet 2018; 392: 866-879.

2 Gaillard EA, Carty H, Heaf D, et al. Reversible bronchial dilatation in children: comparison of serial high-resolution computer tomography scans of the lungs. Eur J Radiol 2003; 47: 215-220. 
3

Flume PA, Chalmers JD, Olivier KN. Advances in bronchiectasis: endotyping, genetics, microbiome, and disease heterogeneity. Lancet 2018; 392: 880-890.

Goyal V, Grimwood K, Masters IB, et al. State of the art: pediatric bronchiectasis. Pediatr Pulmonol 2016; 51 450-469.

Gibson GJ, Loddenkemper R, Sibille Y, et al. Bronchiectasis. In: European Lung White Book. 2nd Edn. Sheffield, European Respiratory Society, 2013; pp. 224-237.

Kapur N, Masters IB, Newcombe P, et al. The burden of disease in pediatric non-cystic fibrosis bronchiectasis. Chest 2012; 141: 1018-1024.

Goyal V, McPhail SM, Hurley F, et al. Cost of hospitalisation for bronchiectasis exacerbations in children. Respirology 2020; 25: 1250-1256.

Cox NS, Wilson CJ, Bennett KA, et al. Health-related quality of life and psychological wellbeing are poor in children with bronchiectasis and their parents. ERJ Open Res 2019; 5: 00063-2019.

Nathan AM, Muthusamy A, Thavagnanam S, et al. Chronic suppurative lung disease in a developing country: impact on child and parent. Pediatr Pulmonol 2014; 49: 435-440.

Prentice BJ, Wales S, Doumit M, et al. Children with bronchiectasis have poorer lung function than those with cystic fibrosis and do not receive the same standard of care. Pediatr Pulmonol 2019; 54: 1921-1926.

McCallum GB, Chang AB. 'Good enough' is 'not enough' when managing Indigenous adults with bronchiectasis in Australia and New Zealand. Respirology 2018; 23: 725-726.

Chalmers JD, Chang AB, Chotirmall SH, et al. Bronchiectasis. Nat Rev Dis Primers 2018; 4: 45.

Chang $A B$, Redding GJ, Everard ML. Chronic wet cough: protracted bronchitis, chronic suppurative lung disease and bronchiectasis. Pediatr Pulmonol 2008; 43: 519-531.

Kapur N, Masters IB, Chang AB. Longitudinal growth and lung function in pediatric non-CF bronchiectasis what influences lung function stability? Chest 2010; 138: 158-164.

Lovie-Toon Y, Grimwood K, Byrnes CA, et al. Health-resource use and quality of life in children with bronchiectasis: a multi-center pilot cohort study. BMC Health Serv Res 2019; 19: 561.

Hill AT, Haworth CS, Aliberti S, et al. Pulmonary exacerbation in adults with bronchiectasis: a consensus definition for clinical research. Eur Respir J 2017; 49: 1700051.

Valery PC, Morris PS, Byrnes CA, et al. Long term azithromycin for Indigenous children with non-cystic fibrosis bronchiectasis or chronic suppurative lung disease (Bronchiectasis Intervention Study): a multi-centre, double-blind randomised controlled trial. Lancet Respir Med 2013; 1: 610-620.

King PT, Holdsworth SR, Farmer M, et al. Phenotypes of adult bronchiectasis: onset of productive cough in childhood and adulthood. COPD 2009; 6: 130-136.

Kapur N, Grimwood K, Masters IB, et al. Lower airway microbiology and cellularity in children with newly diagnosed non-CF bronchiectasis. Pediatr Pulmonol 2012; 47: 300-307.

van der Gast CJ, Cuthbertson L, Rogers GB, et al. Three clinically distinct chronic pediatric airway infections share a common core microbiota. Ann Am Thorac Soc 2014; 11: 1039-1048.

Pizzutto SJ, Yerkovich ST, Upham JW, et al. Children with chronic suppurative lung disease have a reduced capacity to synthesize interferon-gamma in vitro in response to non-typeable Haemophilus influenzae. PLoS One 2014; 9: e104236.

Lee AL, Button BM, Tannenbaum EL. Airway clearance techniques in children and adolescents with chronic suppurative lung disease and bronchiectasis. Front Pediatr 2017; 5: 2.

Polverino E, Goeminne PC, McDonnell MJ, et al. European Respiratory Society guidelines for the management of adult bronchiectasis. Eur Respir J 2017; 50: 1700629.

Hill AT, Sullivan L, Chalmers JD, et al. British Thoracic Society Guideline for bronchiectasis in adults. Thorax 2019; 74: Suppl. 1, 1-69.

Barbato A, Frischer T, Kuehni CE, et al. Primary ciliary dyskinesia: a consensus statement on diagnostic and treatment approaches in children. Eur Respir J 2009; 34: 1264-1276.

Strippoli MP, Frischer T, Barbato A, et al. Management of primary ciliary dyskinesia in European children: recommendations and clinical practice. Eur Respir J 2012; 39: 1482-1491.

Miravitlles M, Tonia T, Rigau D, et al. New era for European Respiratory Society clinical practice guidelines: joining efficiency and high methodological standards. Eur Respir J 2018; 51: 1800221.

Guyatt GH, Oxman AD, Kunz R, et al. GRADE guidelines: 2. Framing the question and deciding on important outcomes. J Clin Epidemiol 2011; 64: 395-400.

Guyatt GH, Oxman AD, Kunz R, et al. Going from evidence to recommendations. BMJ 2008; 336: 1049-1051. Dodd JD, Souza CA, Muller NL. Conventional high-resolution CT versus helical high-resolution MDCT in the detection of bronchiectasis. AJR Am J Roentgenol 2006; 187: 414-420.

1 Hill LE, Ritchie G, Wightman AJ, et al. Comparison between conventional interrupted high-resolution CT and volume multidetector CT acquisition in the assessment of bronchiectasis. Br J Radiol 2010; 83: 67-70.

Matsuoka S, Uchiyama $\mathrm{K}$, Shima $\mathrm{H}$, et al. Bronchoarterial ratio and bronchial wall thickness on high-resolution CT in asymptomatic subjects: correlation with age and smoking. Am J Roentgenol 2003; 180: 513-518. 
Kapur N, Masel JP, Watson D, et al. Bronchoarterial ratio on high resolution CT scan of the chest in children without pulmonary pathology - need to redefine bronchial dilatation. Chest 2011; 139: 1445-1450.

Long FR, Williams RS, Castile RG. Structural airway abnormalities in infants and young children with cystic fibrosis. J Pediatr 2004; 144: 154-161.

Bastardo CM, Sonnappa S, Stanojevic S, et al. Non-cystic fibrosis bronchiectasis in childhood: longitudinal growth and lung function. Thorax 2009; 64: 246-251.

McCallum GB, Singleton RJ, Redding GJ, et al. A decade on: follow-up findings of indigenous children with bronchiectasis. Pediatr Pulmonol 2020; 55: 975-985.

Haidopoulou K, Calder A, Jones A, et al. Bronchiectasis secondary to primary immunodeficiency in children: longitudinal changes in structure and function. Pediatr Pulmonol 2009; 44: 669-675.

Gokdemir Y, Hamzah A, Erdem E, et al. Quality of life in children with non-cystic-fibrosis bronchiectasis. Respiration 2014; 88: 46-51.

Twiss J, Metcalfe R, Edwards EA, et al. New Zealand national incidence of bronchiectasis "too high" for a developed country. Arch Dis Child 2005; 90: 737-740.

Pizzutto SJ, Grimwood K, Bauert P, et al. Bronchoscopy contributes to the clinical management of Indigenous children newly diagnosed with non-cystic fibrosis bronchiectasis. Pediatr Pulmonol 2013; 48: 67-73.

Guran T, Ersu R, Karadag B, et al. Association between inflammatory markers in induced sputum and clinical characteristics in children with non-cystic fibrosis bronchiectasis. Pediatr Pulmonol 2007; 42: 362-369.

Li AM, Sonnappa S, Lex C, et al. Non-CF bronchiectasis: does knowing the aetiology lead to changes in management? Eur Respir J 2005; 26: 8-14.

Chang $A B$, Grimwood $K$, Wilson $A$, et al. Antibiotics for bronchiectasis exacerbations in children: rationale and study protocol for a randomised placebo-controlled trial. Trials 2012; 13: 156.

Chang $\mathrm{AB}$, Grimwood K, Wilson AC, et al. Bronchiectasis Exacerbation Study on azithromycin and amoxycillin-clavulanate for respiratory exacerbations in children (BEST-2): study protocol for a randomized controlled trial. Trials 2013; 14: 53.

Goyal V, Grimwood K, Ware RS, et al. Efficacy of oral antibiotics for non-severe exacerbations of bronchiectasis in children (BEST 1): a multi-centre, double-blind, double-dummy, randomised placebo-controlled trial. Lancet Respir Med 2019; 7: 791-801.

Goyal V, Grimwood K, Byrnes CA, et al. Amoxicillin-clavulanate versus azithromycin for respiratory exacerbations in children with bronchiectasis (BEST-2): a multi-centre, double-blind, non-inferiority randomised controlled trial. Lancet 2018; 392: 1197-1206.

Koh YY, Lee MH, Sun YH, et al. Effect of roxithromycin on airway responsiveness in children with bronchiectasis: a double-blind, placebo-controlled study. Eur Respir J 1997; 10: 994-999.

Masekela R, Anderson R, Gongxeka $\mathrm{H}$, et al. Lack of efficacy of an immunomodulatory macrolide in childhood HIV related bronchiectasis: a randomised, placebo-controlled trial. J Antivir Antiretrovir 2013; 5: 44-49.

Kapur N, Masters IB, Morris PS, et al. Defining pulmonary exacerbation in children with non-cystic fibrosis bronchiectasis. Pediatr Pulmonol 2012; 47: 68-75.

Kapur N, Masters IB, Chang AB. Exacerbations in non cystic fibrosis bronchiectasis: clinical features and investigations. Respir Med 2009; 103: 1681-1687.

Sunther M, Bush A, Hogg C, et al. Recovery of baseline lung function after pulmonary exacerbation in children with primary ciliary dyskinesia. Pediatr Pulmonol 2016; 51: 1362-1366.

Kobbernagel HE, Buchvald FF, Haarman EG, et al. Study protocol, rationale and recruitment in a European multi-centre randomized controlled trial to determine the efficacy and safety of azithromycin maintenance therapy for 6 months in primary ciliary dyskinesia. BMC Pulm Med 2016; 16: 104.

53 Kobbernagel HE, Buchvald FF, Haarman EG, et al. Efficacy and safety of azithromycin maintenance therapy in primary ciliary dyskinesia (BESTCILIA): a multicentre, double-blind, randomised, placebo-controlled phase 3 trial. Lancet Respir Med 2020; 8: 493-505.

Lucas JS, Gahleitner F, Amorim A, et al. Pulmonary exacerbations in patients with primary ciliary dyskinesia: an expert consensus definition for use in clinical trials. ERJ Open Res 2019; 5: 00147-2018.

Shapiro AJ, Zariwala MA, Ferkol T, et al. Diagnosis, monitoring, and treatment of primary ciliary dyskinesia: PCD foundation consensus recommendations based on state of the art review. Pediatr Pulmonol 2016; 51: 115-132. Indinnimeo L, Tancredi G, Barreto M, et al. Effects of a program of hospital-supervised chest physical therapy on lung function tests in children with chronic respiratory disease: 1-year follow-up. Int $J$ Immunopathol Pharmacol 2007; 20: 841-845.

Murray MP, Pentland JL, Hill AT. A randomised crossover trial of chest physiotherapy in non-cystic fibrosis bronchiectasis. Eur Respir J 2009; 34: 1086-1092.

Munoz G, de Gracia J, Buxo M, et al. Long-term benefits of airway clearance in bronchiectasis: a randomised placebo-controlled trial. Eur Respir J 2018; 51: 1701926. 
Hill AT, Barker AF, Bolser DC, et al. Treating cough due to non-CF and CF bronchiectasis with nonpharmacological airway clearance: CHEST Expert Panel Report. Chest 2018; 153: 986-993.

Lee AL, Williamson HC, Lorensini S, et al. The effects of oscillating positive expiratory pressure therapy in adults with stable non-cystic fibrosis bronchiectasis: a systematic review. Chron Respir Dis 2015; 12: 36-46.

Phillips J, Lee A, Pope R, et al. Effect of airway clearance techniques in patients experiencing an acute exacerbation of bronchiectasis: a systematic review. Physiother Theory Pract 2020; 36: 1300-1315.

Warnock L, Gates A. Chest physiotherapy compared to no chest physiotherapy for cystic fibrosis. Cochrane Database Syst Rev 2015; 12: CD001401.

Button BM, Wilson C, Dentice R, et al. Physiotherapy for cystic fibrosis in Australia and New Zealand: a clinical practice guideline. Respirology 2016; 21: 656-667.

Wilson LM, Morrison L, Robinson KA. Airway clearance techniques for cystic fibrosis: an overview of Cochrane systematic reviews. Cochrane Database Syst Rev 2019; 1: CD011231.

Desmond KJ, Schwenk WF, Thomas E, et al. Immediate and long-term effects of chest physiotherapy in patients with cystic fibrosis. J Pediatr 1983; 103: 538-542.

O'Donnell AE, Barker AF, llowite JS, et al. Treatment of idiopathic bronchiectasis with aerosolized recombinant human DNase I. Chest 1998; 113: 1329-1334.

Wills PJ, Wodehouse T, Corkery K, et al. Short-term recombinant human DNase in bronchiectasis. Am J Respir Crit Care Med 1996; 154: 413-417.

Paff T, Daniels JM, Weersink EJ, et al. A randomised controlled trial on the effect of inhaled hypertonic saline on quality of life in primary ciliary dyskinesia. Eur Respir J 2017; 49: 1601770.

Nicolson CH, Stirling RG, Borg BM, et al. The long term effect of inhaled hypertonic saline $6 \%$ in non-cystic fibrosis bronchiectasis. Respir Med 2012; 106: 661-667.

Kellett F, Robert NM. Nebulised 7\% hypertonic saline improves lung function and quality of life in bronchiectasis. Respir Med 2011; 105: 1831-1835.

Bilton D, Tino G, Barker AF, et al. Inhaled mannitol for non-cystic fibrosis bronchiectasis: a randomised, controlled trial. Thorax 2014; 69: 1073-1079.

Bilton D, Daviskas E, Anderson SD, et al. Phase 3 randomized study of the efficacy and safety of inhaled dry powder mannitol for the symptomatic treatment of non-cystic fibrosis bronchiectasis. Chest 2013; 144: 215-225.

Olivieri D, Ciaccia A, Marangio E, et al. Role of bromhexine in exacerbations of bronchiectasis. Double-blind randomized multicenter study versus placebo. Respiration 1991; 58: 117-121.

Gao YH, Abo LH, Finch S, et al. The relationship between symptoms, exacerbations and treatment response in bronchiectasis. Am J Respir Crit Care Med 2020; 201: 1499-1507.

Wang D, Fu W, Dai J. Meta-analysis of macrolide maintenance therapy for prevention of disease exacerbations in patients with noncystic fibrosis bronchiectasis. Medicine 2019; 98: e15285.

Milito C, Pulvirenti F, Cinetto F, et al. Double-blind, placebo-controlled, randomized trial on low-dose azithromycin prophylaxis in patients with primary antibody deficiencies. J Allergy Clin Immunol 2019; 144: 584-593.

Hare KM, Grimwood K, Chang AB, et al. Nasopharyngeal carriage and macrolide resistance in Indigenous children with bronchiectasis randomized to long-term azithromycin or placebo. Eur J Clin Microbiol Infect Dis 2015; 34: 2275-2285.

Orriols R, Hernando R, Ferrer A, et al. Eradication therapy against Pseudomonas aeruginosa in non-cystic fibrosis bronchiectasis. Respiration 2015; 90: 299-305.

White L, Mirrani G, Grover M, et al. Outcomes of Pseudomonas eradication therapy in patients with non-cystic fibrosis bronchiectasis. Respir Med 2012; 106: 356-360.

Blanco-Aparicio M, Saleta Canosa JL, Valino LP, et al. Eradication of Pseudomonas aeruginosa with inhaled colistin in adults with non-cystic fibrosis bronchiectasis. Chron Respir Dis 2019; 16: 1479973119872513.

Langton Hewer SC, Smyth AR. Antibiotic strategies for eradicating Pseudomonas aeruginosa in people with cystic fibrosis. Cochrane Database Syst Rev 2017; 4: CD004197.

Lo DK, Hurley MN, Muhlebach MS, et al. Interventions for the eradication of methicillin-resistant Staphylococcus aureus (MRSA) in people with cystic fibrosis. Cochrane Database Syst Rev 2013; 2: CD009650.

Laska IF, Crichton ML, Shoemark A, et al. The efficacy and safety of inhaled antibiotics for the treatment of bronchiectasis in adults: a systematic review and meta-analysis. Lancet Respir Med 2019; 7: 855-869.

8 Lim JU, Hong SW, Ko JH. Efficacy of inhaled ciprofloxacin agents for the treatment of bronchiectasis: a systematic review and meta-analysis of randomized controlled trials. Ther Adv Respir Dis 2019; 13: 1753466619875930.

Araujo D, Shteinberg M, Aliberti S, et al. The independent contribution of Pseudomonas aeruginosa infection to long-term clinical outcomes in bronchiectasis. Eur Respir J 2018; 51: 1701953.

Doring G, Conway SP, Heijerman HG, et al. Antibiotic therapy against Pseudomonas aeruginosa in cystic fibrosis: a European consensus. Eur Respir J 2000; 16: 749-767.

Nixon GM, Armstrong DS, Carzino R, et al. Clinical outcome after early Pseudomonas aeruginosa infection in cystic fibrosis. J Pediatr 2001; 138: 699-704. 
Guran T, Ersu R, Karadag B, et al. Withdrawal of inhaled steroids in children with non-cystic fibrosis bronchiectasis. J Clin Pharm Ther 2008; 33: 603-611.

89 Kapur N, Petsky HL, Bell S, et al. Inhaled steroids for bronchiectasis. Cochrane Database Syst Rev 2018; 5: CD000996.

90 Goyal V, Chang AB. Combination inhaled corticosteroids and long-acting beta2-agonists for children and adults with bronchiectasis. Cochrane Database Syst Rev 2014; 6: CD010327.

91 Hernando R, Drobnic ME, Cruz MJ, et al. Budesonide efficacy and safety in patients with bronchiectasis not due to cystic fibrosis. Int J Clin Pharm 2012; 34: 644-650.

92 Tsang KW, Tan KC, Ho PL, et al. Inhaled fluticasone in bronchiectasis: a 12 month study. Thorax 2005; 60: 239-243.

93 Tsang KW, Ho PL, Lam WK, et al. Inhaled fluticasone reduces sputum inflammatory indices in severe bronchiectasis. Am J Respir Crit Care Med 1998; 158: 723-727.

94 Martinez-Garcia MA, Perpina-Tordera M, Roman-Sanchez P, et al. Inhaled steroids improve quality of life in patients with steady-state bronchiectasis. Respir Med 2006; 100: 1623-1632.

95 Martinez-Garcia MA, Soler-Cataluna JJ, Catalan-Serra P, et al. Clinical efficacy and safety of budesonideformoterol in non-cystic fibrosis bronchiectasis. Chest 2012; 141: 461-468.

96 Andrejak C, Nielsen R, Thomsen VO, et al. Chronic respiratory disease, inhaled corticosteroids and risk of non-tuberculous mycobacteriosis. Thorax 2013; 68: 256-262.

97 Lee $\mathrm{CH}$, Kim K, Hyun MK, et al. Use of inhaled corticosteroids and the risk of tuberculosis. Thorax 2013; 68: 1105-1113.

98 Cavkaytar O, Vuralli D, Arik YE, et al. Evidence of hypothalamic-pituitary-adrenal axis suppression during moderate-to-high-dose inhaled corticosteroid use. Eur J Pediatr 2015; 174: 1421-1431.

99 Loke YK, Blanco P, Thavarajah M, et al. Impact of inhaled corticosteroids on growth in children with asthma: systematic review and meta-analysis. PLoS One 2015; 10: e0133428.

100 Todd GR, Acerini CL, Ross-Russell R, et al. Survey of adrenal crisis associated with inhaled corticosteroids in the United Kingdom. Arch Dis Child 2002; 87: 457-461.

101 Leung JS, Johnson DW, Sperou AJ, et al. A systematic review of adverse drug events associated with administration of common asthma medications in children. PLOS One 2017; 12: e0182738.

102 Henkle E, Curtis JR, Chen L, et al. Comparative risks of chronic inhaled corticosteroids and macrolides for bronchiectasis. Eur Respir J 2019; 54: 1801896.

103 Al-Kattan KM, Essa MA, Hajjar WM, et al. Surgical results for bronchiectasis based on hemodynamic (functional and morphologic) classification. J Thorac Cardiovasc Surg 2005; 130: 1385-1390.

104 Fan LC, Liang S, Lu HW, et al. Efficiency and safety of surgical intervention to patients with non-cystic fibrosis bronchiectasis: a meta-analysis. Sci Rep 2015; 5: 17382.

105 Lee AL, Hill CJ, Cecins N, et al. The short and long term effects of exercise training in non-cystic fibrosis bronchiectasis - a randomised controlled trial. Respir Res 2014; 15: 44.

106 Dona E, Olveira C, Palenque FJ, et al. Pulmonary rehabilitation only versus with nutritional supplementation in patients with bronchiectasis: a randomised controlled trial. J Cardiopulm Rehabil Prev 2018; 38: 411-418.

107 O'Grady KF, Chang AB, Cripps A, et al. The clinical, immunological and microbiological impact of the 10 -valent pneumococcal-Protein $D$ conjugate vaccine in children with recurrent protracted bacterial bronchitis, chronic suppurative lung disease and bronchiectasis: a multi-centre, double-blind, randomised controlled trial. Hum Vaccin Immunother 2018; 14: 2768-2779.

108 Mirra V, Caffarelli C, Maglione M, et al. Hypovitaminosis D: a novel finding in primary ciliary dyskinesia. Ital J Pediatr 2015; 41: 14.

109 Lavery K, O'Neill B, Elborn JS, et al. Self-management in bronchiectasis: the patients' perspective. Eur Respir J 2007; 29: 541-547.

110 Chang CC, Singleton RJ, Morris PS, et al. Pneumococcal vaccines for children and adults with bronchiectasis. Cochrane Database Syst Rev 2009; 2: CD006316.

111 Chalmers JD, McHugh BJ, Docherty C, et al. Vitamin-D deficiency is associated with chronic bacterial colonisation and disease severity in bronchiectasis. Thorax 2013; 68: 39-47.

112 Alanin MC, Nielsen KG, von BC, et al. A longitudinal study of lung bacterial pathogens in patients with primary ciliary dyskinesia. Clin Microbiol Infect 2015; 21: 1093-1097.

113 Cohen-Cymberknoh M, Weigert N, Gileles-Hillel A, et al. Clinical impact of Pseudomonas aeruginosa colonization in patients with primary ciliary dyskinesia. Respir Med 2017; 131: 241-246.

114 Hare KM, Chang AB, Smith-Vaughan HC, et al. Do combined upper airway cultures identify lower airway infections in children with chronic cough? Pediatr Pulmonol 2019; 54: 907-913.

115 Munro KA, Reed PW, Joyce H, et al. Do New Zealand children with non-cystic fibrosis bronchiectasis show disease progression? Pediatr Pulmonol 2011; 46: 131-138.

116 Chalmers JD, Ringshausen FC, Harris B, et al. Cross-infection risk in patients with bronchiectasis: a position statement from the European Bronchiectasis Network (EMBARC), EMBARC/ELF patient advisory group and European Reference Network (ERN-Lung) Bronchiectasis Network. Eur Respir J 2018; 51: 1701937. 
117 Bilan N, Aghakhani M, Niafar F. Factors affecting the outcome of bronchiectasis in pediatric patients. Int $J$ Pediatr 2014; 2: 377-389.

118 Eastham KM, Fall AJ, Mitchell L, et al. The need to redefine non-cystic fibrosis bronchiectasis in childhood. Thorax 2004; 59: 324-327.

119 Banjar HH. Clinical profile of Saudi children with bronchiectasis. Indian J Pediatr 2007; 74: 149-152.

120 Maglione M, Bush A, Montella S, et al. Progression of lung disease in primary ciliary dyskinesia: is spirometry less accurate than CT? Pediatr Pulmonol 2012; 47: 498-504.

121 Magnin ML, Cros P, Beydon N, et al. Longitudinal lung function and structural changes in children with primary ciliary dyskinesia. Pediatr Pulmonol 2012; 47: 816-825.

122 Manson D, Reid B, Dalal I, et al. Clinical utility of high-resolution pulmonary computed tomography in children with antibody deficiency disorders. Pediatr Radiol 1997; 27: 794-798.

123 Redding GJ, Singleton RJ, Valery PC, et al. Respiratory exacerbations in indigenous children from two countries with non-cystic fibrosis chronic suppurative lung disease/bronchiectasis. Chest 2014; 146: 762-774.

124 Brenner DJ. Estimating cancer risks from pediatric CT: going from the qualitative to the quantitative. Pediatr Radiol 2002; 32: 228-231.

125 Baris S, Ercan H, Cagan HH, et al. Efficacy of intravenous immunoglobulin treatment in children with common variable immunodeficiency. J Investig Allergol Clin Immunol 2011; 21: 514-521.

126 Crowley S, Matthews I. Resolution of extensive severe bronchiectasis in an infant. Pediatr Pulmonol 2010; 45: 717-720.

127 Mansour $\mathrm{Y}$, Beck R, Danino J, et al. Resolution of severe bronchiectasis after removal of long-standing retained foreign body. Pediatr Pulmonol 1998; 25: 130-132.

128 Mallick MS. Tracheobronchial foreign body aspiration in children: a continuing diagnostic challenge. Afr J Paediatr Surg 2014; 11: 225-228.

129 Karakoc F, Karadag B, Akbenlioglu C, et al. Foreign body aspiration: what is the outcome? Pediatr Pulmonol 2002; 34: 30-36.

130 Byrnes CA, Trenholme A, Lawrence S, et al. Prospective community programme versus parent-driven care to prevent respiratory morbidity in children following hospitalisation with severe bronchiolitis or pneumonia. Thorax 2020; 75: 298-305.

131 Valery PC, Torzillo PJ, Mulholland EK, et al. A hospital-based case-control study of bronchiectasis in Indigenous children in Central Australia. Pediatr Infect Dis J 2004; 23: 902-908.

132 Singleton RJ, Valery PC, Morris P, et al. Indigenous children from three countries with non-cystic fibrosis chronic suppurative lung disease/bronchiectasis. Pediatr Pulmonol 2014; 49: 189-200.

133 Wurzel DF, Marchant JM, Yerkovich ST, et al. Protracted bacterial bronchitis in children: natural history and risk factors for bronchiectasis. Chest 2016; 150: 1101-1108.

134 Moberg J, Oxman AD, Rosenbaum S, et al. The GRADE Evidence to Decision (EtD) framework for health system and public health decisions. Health Res Policy Syst 2018; 16: 45. 